\title{
COBERTURA NIVAL Y DISTRIBUCIÓN DE LA TEMPERATURA EN EL SUELO EN LAS CUMBRES DE LA SIERRA DE GUADARRAMA
}

\author{
N. ANDRÉS DE PABLO y D. PALACIOS ESTREMERA \\ Dpto. de Análisis Geográfico Regional y Geografía Física \\ Universidad Complutense de Madrid \\ Correo electrónico de contacto: nuriand@ghis.ucm.es
}

\begin{abstract}
RESUMEN. El trabajo muestra el resultado de la monitorización de la temperatura del aire y del suelo en el período 2002-2007, en las proximidades de la cumbre del Alto de las Guarramillas (2.258 m; 40 47' 10', $N$ y $3^{\circ} 58^{\prime} 46^{\prime}$ ' W), situada en el sector central de la Sierra de Guadarrama, a 50 km de Madrid. La temperatura del aire se midió en la cumbre y la del suelo en tres lugares distintos, según el diferente grado de permanencia de la nieve en el suelo: en la cumbre, en el interior de un nicho nival situado en la vertiente oriental de esta cumbre, el Ventisquero de la Condesa, y en el sector pronival de este nicho. Por otro lado, se monitorizó la permanencia y espesor de la nieve sobre cada termómetro del suelo. Los resultados indican que, mientras la temperatura estival del suelo es idéntica en los tres puntos de control y simétrica en sus cambios a la del aire, durante la temporada nival la temperatura del suelo varía de forma considerable según el punto de medición, en relación con el espesor y duración de la nieve que soporte y puede no tener ninguna relación con la evolución de la temperatura del aire. A la hora de considerar la importancia de los cambios de fase del agua contenida en el suelo sobre los procesos geomorfológicos o sobre la cubierta vegetal, es fundamental el tener en cuenta el espesor y, sobre todo, la duración de la nieve, ya que este factor puede ocasionar el cambio brusco del régimen térmico del suelo en sólo unos pocos metros de diferencia.
\end{abstract}

ABSTRACT. Air and ground temperatures were monitored from 2002-2007 at the summit area of Alto de las Guarramillas (2258 m; 40 47' 10' ' $\mathrm{N}$ and $3^{\circ} 58^{\prime} 46^{\prime \prime}$ $W$ ) in the central sector of Sierra de Guadarrama, a mountain range located $50 \mathrm{~km}$ from Madrid. Air temperature was recorded at the summit while ground temperature was monitored at 3 locations, differentiated by the permanence of snow cover: the summit, inside a snow hollow, known as Ventisquero de la Condesa, located on the east side of the summit and the pronival sector of this hollow. During the summer, ground temperatures were uniform for all three control 
points, and showed symmetrical changes with the air temperature. During the period of snow cover, ground temperature varied widely among the sample sites as influenced by snow thickness and, particularly, snow cover duration. It is uncertain as to whether these variations could be related to variations in air temperature. Snow thickness and snow cover duration are factors that directly affect phase changes in ground water content, which subsequently influences geomorphologic processes and plant cover. Snow cover duration is a crucial factor, because it can markedly alter ground temperature in a distance of only a few meters.

Palabras clave: Sierra de Guadarrama, permanencia de la cubierta de nieve, nicho nival, temperatura del suelo, nivación.

Key words: Sierra de Guadarrama, snow cover duration, snow hollow, ground temperature, nivation.

Enviado el 20 de noviembre de 2009

Aceptado el 19 de febrero de 2010

\section{Introducción}

Desde mediados del siglo XIX la cubierta nival ya fue considerada un factor clave en la distribución el régimen de la temperatura del suelo, al enfrentarse a problemas relacionados con la construcción de infraestructuras en el subsuelo (Rozet, 1855). Sin embargo, a la hora de aplicar este criterio a la geomorfología, se tardó mucho en comprender que la nieve amortiguaba drásticamente la amplitud térmica del suelo. Fue Matthes (1900) el primero que propuso la existencia de neveros permanentes o de larga duración como agentes erosivos. Hobbs (1910) recoge esta idea, y propone que esa capacidad erosiva de los neveros se basa en la abundancia de agua de deshielo circulando bajo la nieve y la proximidad de la temperatura de la superficie de la base del nevero a los $0^{\circ} \mathrm{C}$ durante gran parte del año, lo que indudablemente facilitaría un alto número de ciclos de hielo/deshielo, con respecto a las áreas fuera del nevero. Grawe (1936) inicia la monitorización de las temperaturas para comprobar esa eficacia. Pero no es hasta mucho después, cuando la monitorización térmica del suelo en áreas nivales se generaliza, con trabajos pioneros y claves, como los de Gardener (1969), Thorn (1976 y 1979), Hall (1980) y Thorn y Hall (1980). Estos trabajos revelaron la poca o nula actividad geomorfológica derivada por el hielo/deshielo que existía bajo los neveros, donde el aislamiento de la nieve reducía drásticamente la amplitud térmica en el suelo. Posteriormente se sucedieron trabajos de monitorización de la temperatura del suelo en neveros con detallados seguimientos de varios años, tratando de analizar las condiciones geomorfológicas, derivadas de este régimen térmico, como los publicados por Goodrich (1982), Hall (1985, 1993), Nyberg (1991), Razcokowska (1995), Christiansen (1996), Kariya (2002), e Ishikawa (2003), que confirmaron la poca o nula eficacia de la acción de la helada bajo los neveros. Importantes trabajos de síntesis destacaron la poca fiabilidad de criterios derivados del análisis de la temperatura del aire, aplicados a extrapolar la del suelo, sin tener en cuenta la cubierta nival (Hall et al., 2002). En España, la investiga- 
ción sobre el régimen térmico del suelo bajo los neveros y sus posibles consecuencias geomorfológicas y ecológicas ha atraído poca atención de los científicos (Marcos y Palacios, 2004; Andrés et al. 2007a, Muñoz et al. 2007), con resultados todavía incipientes.

El objetivo de este trabajo es realizar un seguimiento prolongado en el tiempo de la temperatura del aire y del suelo, así como de la duración y espesor de la nieve en los lugares de muestreo de la temperatura del suelo, en un área representativa del centro de la península Ibérica. De esta manera, se podrán comparar los posibles regímenes térmicos del suelo en puntos que presentan diferencias en las características de su cubierta nival.

\section{2. Área de estudio y métodos}

Para realizar la experimentación propuesta se ha elegido la Sierra de Guadarrama, justo en una de sus cumbres situada en el sector central de la misma, Alto de las Guarramillas (2.258 m; $40^{\circ} 47^{\prime} 10^{\prime}$ ' $\mathrm{N}$ y $3^{\circ} 58^{\prime} 46^{\prime}$ ' W), a $50 \mathrm{~km}$ al norte de la ciudad de Madrid (Fig. 1). Aprovechando una infraestructura de comunicaciones situada justo en la cima, se pudo instalar un termómetro automático del aire, denominado AIRE (2.255 m; 40 47' 5' $\mathrm{N}$ y 3 58' 46' W), a 1 m del suelo, desde el año 2002 al año 2007, el cual funcionó de forma correcta salvo el primer año y, posteriormente, en un período de 3 meses, que no coincidió con el invierno.

Inmediatamente al sureste del Alto de las Guarramillas se localiza un nevero de larga duración, conocido como Ventisquero de la Condesa (Fig. 2), donde, en su sector

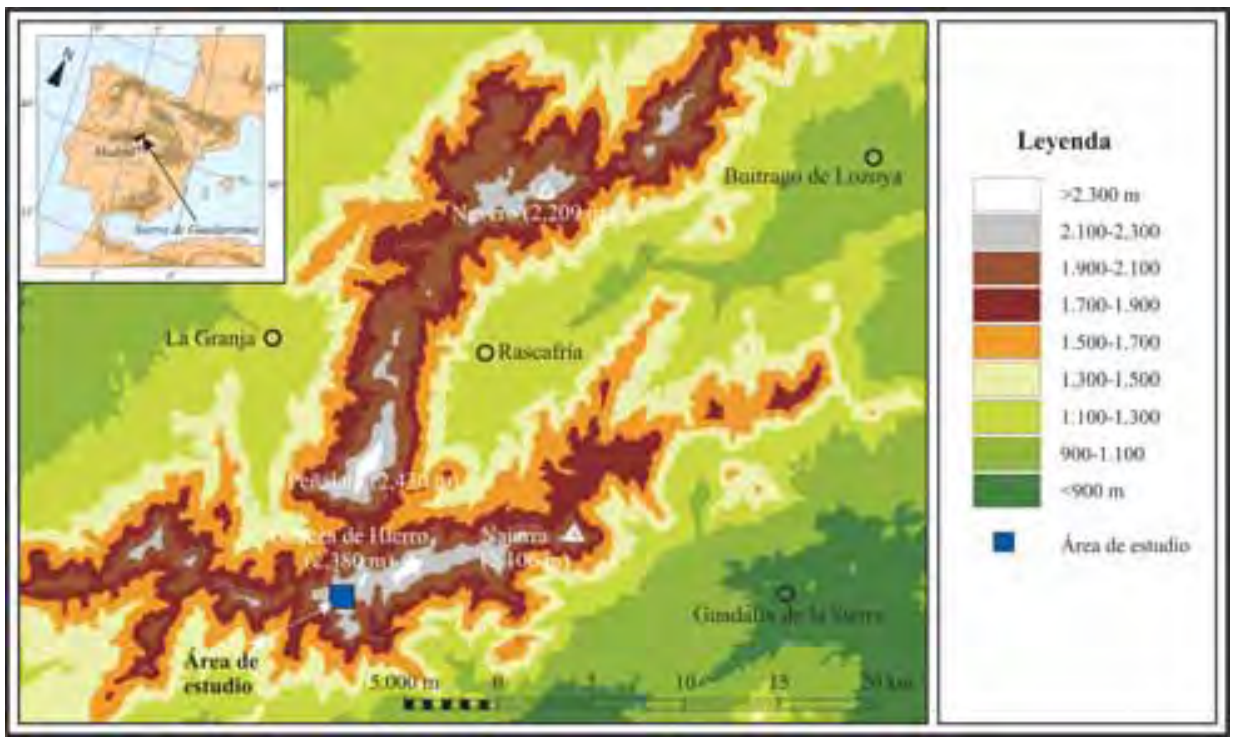

Figura 1. Situación del área de estudio, el Alto de las Guarramillas, en el centro de la Sierra de Guadarrama. 


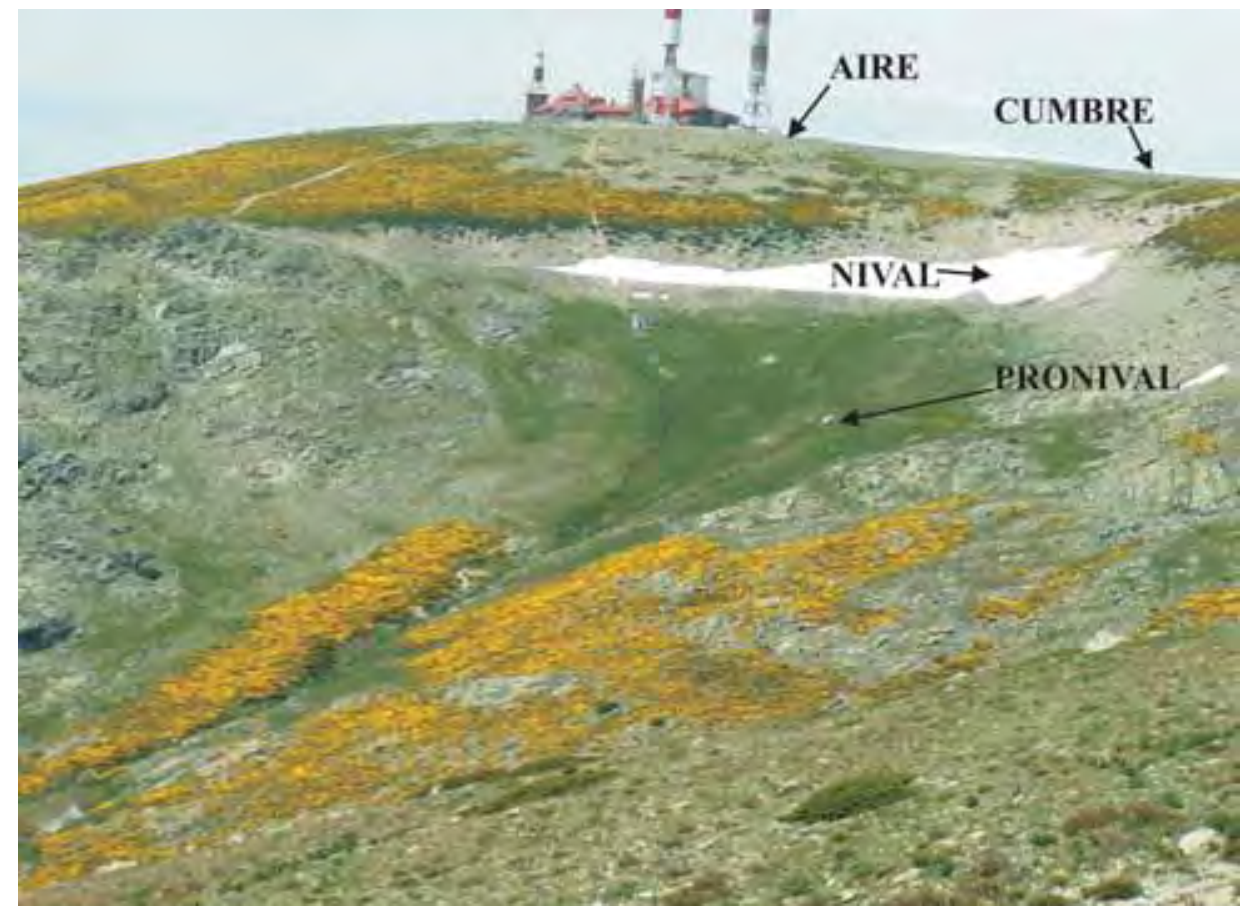

Figura 2. Foto del Alto de las Guarramillas y del Ventisquero de la Condesa, donde se indica la localización de las estaciones térmicas.

central, la nieve permanece más de 200 días al año y cuyas características geomorfológicas y biogeográficas son bien conocidas (Andrés y Palacios 2004; Muñoz y GarcíaRomero 2004, Andrés et al. 2007b, García-Romero et al. 2009). Por el contrario, la cumbre, muy venteada, permanece sin nieve gran parte del invierno. Una tercera situación es la que ofrece el área adyacente al frente del nevero, en una posición topográfica más baja que éste, que, a pesar de carecer de una cubierta nival duradera, se ve afectada por las aguas de deshielo durante toda la primavera y hasta bien entrado el verano. Estas circunstancias se vieron como las idóneas para una mayor hidratación del suelo y una mayor eficacia de los ciclos de hielo/deshielo, lo que llevaría a explicar cómo frecuentemente las áreas pronivales están ocupadas por grandes coladas de gelifluxión, hecho ya resaltado desde los primeros estudios en nivación (Ekblaw, 1918), y que también ocurre en el Ventisquero de la Condesa.

Siguiendo las pautas de estas observaciones, se seleccionaron tres puntos para localizar los termómetros del suelo, con el máximo contraste en relación con la cubierta nival (Fig. 3). En cada estación se instalaron dos sensores, uno situado a $10 \mathrm{~cm}$ de profundidad en el suelo y otro situado a $60 \mathrm{~cm}$. Las estaciones estuvieron en funcionamiento desde el año 2002 al 2007, aunque en algunos casos se perdieron los sensores o estos no recogieron correctamente los datos. Se utilizaron sondas HOBO Pendant $64 \mathrm{~K}$ 


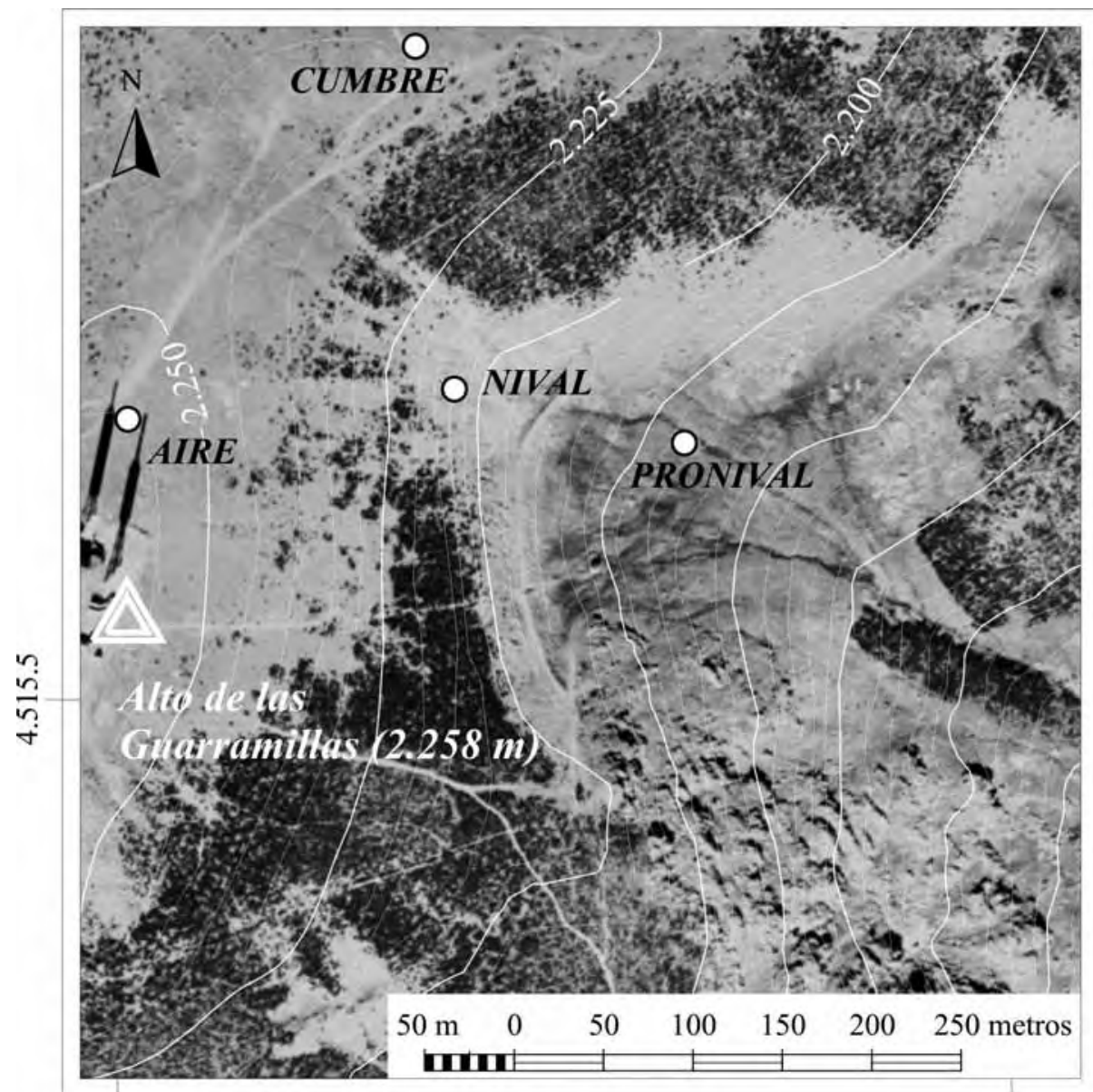

$417.500 \mathrm{~m}$

418.0

Figura 3. Ortofoto y mapa del Ventisquero de la Condesa, con la localización de las estaciones térmicas.

Temp/Light, dotadas de termistores TMC-1T y encapsuladas en una envoltura impermeable. Los termómetros están equipados con una batería de litio de $3.6 \mathrm{~V}$, con capacidad para tomar medidas durante 760 días cada 30 minutos, y tienen una precisión de $+/-0,1^{\circ} \mathrm{C}$, y un rango de $-20^{\circ} \mathrm{C}$ a $+50^{\circ} \mathrm{C}$.

La primera estación, denominada CUMBRE (2.243 m; 40 47' $14^{\prime \prime}$ ' $\mathrm{N}$ y $3^{\circ} 58^{\prime} 40^{\prime \prime}$ W), se situó a $250 \mathrm{~m}$ al NE de la cima del Alto de las Guarramillas, en el sector más ventoso de la cresta y donde la nieve permanece un menor número de días al año. Se pudieron usar los datos de esta estación de todos los años, salvo el último. Una segunda estación del suelo, denominada NIVAL ( $2.212 \mathrm{~m}$; $40^{\circ} 47^{\prime} 10^{\prime \prime} \mathrm{N}$ y $\left.3^{\circ} 58^{\prime} 35^{\prime \prime} \mathrm{W}\right)$, se situó a $190 \mathrm{~m}$ al SE de la estación CUMBRE, justo en el centro del Ventisquero, allí 
donde la nieve permanece más tiempo sobre el suelo. De esta estación se pudieron analizar todos los datos, excepto la primera temporada del sensor a $-60 \mathrm{~cm}$. La última estación, llamada PRONIVAL (2.163 m; 40 47' 55' ' N y 3 58' 29'” W), se situó a $130 \mathrm{~m}$ al E de la estación NIVAL, en el área pronival, en una de las coladas de solifluxión que nacen en el frente del nevero, pero fuera ya del sector que soporta un elevado número de días con nieve al año. Excepcionalmente, el sensor más profundo se localizó a $-80 \mathrm{~cm}$ en vez de a -60 como en los otros casos. De esta estación se perdió la temporada 2005/2006 entera, al ser destruida por unos excursionistas. Del sensor a $-80 \mathrm{~cm}$ sólo se pudo aprovechar la temporada 2004/05.

En un primer lugar se depuraron los registros obtenidos con las sondas térmicas, y se desecharon series enteras por sus valores erróneos o inseguros, con lo que se lograron series de datos mucho más limitadas de lo esperado en un principio, pero de alto valor cualitativo. Con estos datos, tratados en una hoja de cálculo, se obtuvieron los estadísticos diarios (temperatura media verdadera, mínima y máxima absoluta y amplitud térmica diaria) y mensuales (temperatura media). En segundo lugar, se calcularon el número de días con temperatura mínima superior a $0^{\circ} \mathrm{C}$, los días con temperatura máxima con valores inferiores a $0^{\circ} \mathrm{C}$ y los días donde la temperatura osciló por encima y por debajo de $\operatorname{los} 0^{\circ} \mathrm{C}$. Ante la homogeneidad de los datos entre todas las estaciones de los meses de verano y teniendo en cuenta el objetivo del trabajo, sólo se consideraron los datos de los meses correspondientes a la estación nival, es decir, de octubre a junio.

Por otro lado, se instaló una pértiga reglada en cada estación de $2 \mathrm{~m}$ de altura. Con ayuda de los guardas de medioambiente de la Comunidad de Madrid y un grupo de voluntarios, se pudo obtener medidas del espesor de la nieve, aproximadamente una vez por semana. En la estación NIVAL, la pértiga estuvo tapada por la nieve durante varios períodos, para los que se estimaron espesores máximos entre 4 y 6 m, en los distintos años muestreados.

\section{Resultados}

\subsection{Temperatura del aire}

Los resultados del seguimiento de la temperatura del aire, en la estación AIRE (Fig. 4 y Tablas 1, 2, 3 y 4) muestran una cierta regularidad en el comportamiento del régimen térmico durante la estación nival en los cuatro años de observación. La media mensual (Tabla 1) es negativa o muy próxima a $0^{\circ} \mathrm{C}$ de enero a marzo. Los meses más fríos pueden variar entre diciembre y febrero a lo largo de los años. Las temperaturas mínimas absolutas (Tabla 2) son negativas de octubre a mayo, de hecho, no se ha dado ningún día con temperatura negativa desde julio a septiembre en todo el período de observación. Las mínimas anuales absolutas se pueden producir entre diciembre y marzo, llegando a temperaturas mínimas que oscilan entre $-12 \mathrm{a}-15^{\circ} \mathrm{C}$, si exceptuamos el cálido invierno del 2006/07. Las temperaturas medias diarias (Tabla 3) indican que las mínimas medias diarias se dan en diciembre y enero, aunque las de los meses de febrero y marzo tienen valores muy próximos. La media mensual de la amplitud térmica diaria (Tabla 4) oscila entre 3 y $12^{\circ} \mathrm{C}$ y la oscilación diaria más elevada se produce siempre durante el mes de junio. 


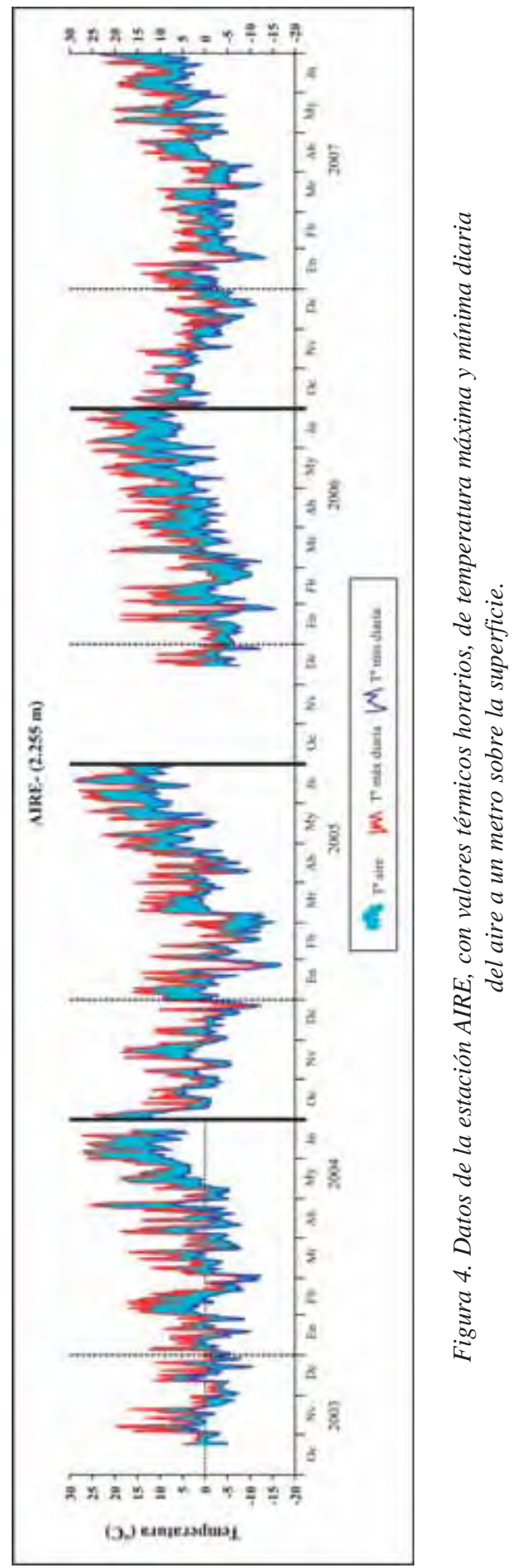


Tabla 1. Temperaturas medias mensuales del aire obtenidas en el sensor AIRE (2.255 m s.n.m.). (*) Sólo se emplean los 22 primeros días de junio de 2004. (**) Faltan los 14 primeros días de diciembre de 2005. (***) Faltan los dos últimos días de octubre de 2006.

\begin{tabular}{|c|c|c|c|c|}
\hline \multicolumn{5}{|c|}{ TEMPERATURA MEDIA MENSUAL: ESTACIÓN AIRE } \\
\hline MES & 2003-04 & 2004-05 & 2005-06 & 2006-07 \\
\hline OCT & & 5,29 & & $6,15(* * *)$ \\
\hline NOV & 2,14 & 2,83 & & 2,89 \\
\hline DIC & $-1,22$ & $-1,83$ & $-1,96(* *)$ & $-2,12$ \\
\hline ENE & $-0,70$ & $-0,80$ & $-3,43$ & $-0,17$ \\
\hline FEB & 0,29 & $-4,83$ & $-1,90$ & $-0,98$ \\
\hline MAR & $-0,13$ & 0,25 & 0,71 & $-1,29$ \\
\hline $\mathrm{ABR}$ & 2,17 & 2,23 & 4,14 & 1,60 \\
\hline MAY & 4,92 & 8,95 & 8,88 & 5,12 \\
\hline JUN & $13,83(*)$ & 14,81 & 13,00 & 9,69 \\
\hline
\end{tabular}

Tabla 2. Temperaturas máximas y mínimas absolutas mensuales registradas por el termómetro AIRE (2.255 m s.n.m.).

\begin{tabular}{|c|c|c|c|c|c|c|c|c|}
\hline \multicolumn{9}{|c|}{ TEMPERATURA HORARIA ABSOLUTA DE LA ESTACIÓN AIRE } \\
\hline \multirow{2}{*}{ MES } & \multicolumn{2}{|c|}{ 2003-04 } & \multicolumn{2}{|c|}{ 2004-05 } & \multicolumn{2}{|c|}{ 2005-06 } & \multicolumn{2}{|c|}{ 2006-07 } \\
\hline & $M A X$ & $M I N$ & $M A X$ & MIN & $\boldsymbol{M A X}$ & MIN & MAX & MIN \\
\hline$\overline{\mathrm{OCT}}$ & & & 25,51 & $-3,87$ & & & 15,72 & $-0,97$ \\
\hline NOV & 19,34 & $-6,30$ & 18,48 & $-5,86$ & & & 14,66 & $-5,63$ \\
\hline DIC & 11,09 & $-10,58$ & 10,84 & $-12,42$ & 10,84 & $-12,18$ & 11,09 & $-11,03$ \\
\hline ENE & 12,34 & $-10,12$ & 15,99 & $-16,68$ & 18,77 & $-15,72$ & 15,45 & $-13,34$ \\
\hline FEB & 17,36 & $-11,72$ & 11,84 & $-13,34$ & 18,19 & $-10,35$ & 7,46 & $-6,97$ \\
\hline MAR & 16,80 & $-12,42$ & 15,45 & $-15,23$ & 20,81 & $-12,42$ & 10,34 & $-12,88$ \\
\hline $\mathrm{ABR}$ & 25,51 & $-7,86$ & 19,93 & $-9,67$ & 18,77 & $-4,31$ & 14,66 & $-10,35$ \\
\hline MAY & 25,84 & $-5,41$ & 25,84 & $-2,53$ & 24,85 & $-4,09$ & 19,93 & $-5,19$ \\
\hline JUN & 27,20 & 3,97 & 28,61 & 3,74 & 26,18 & $-1,87$ & 23,24 & $-1,20$ \\
\hline
\end{tabular}

Tabla 3. Media mensual de las temperaturas máxima y mínima diarias registradas por el termómetro AIRE (2.255 m s.n.m.).

\begin{tabular}{|c|c|c|c|c|c|c|c|c|}
\hline \multicolumn{9}{|c|}{ TEMPERATURA MEDIA DIARIA } \\
\hline \multirow{2}{*}{ MES } & \multicolumn{2}{|c|}{ 2003-04 } & \multicolumn{2}{|c|}{ 2004-05 } & \multicolumn{2}{|c|}{ 2005-06 } & \multicolumn{2}{|c|}{ 2006-07 } \\
\hline & MAX & MIN & $M A X$ & $M I N$ & $M A X$ & MIN & $M A X$ & MIN \\
\hline OCT & & & 9,10 & 2,98 & & & 9,53 & 3,44 \\
\hline NOV & 5,84 & $-0,29$ & 7,66 & 0,20 & & & 5,75 & 0,72 \\
\hline DIC & 1,71 & $-3,33$ & 1,19 & $-4,06$ & 2,41 & $-5,09$ & 1,66 & $-4,83$ \\
\hline ENE & 2,75 & $-3,02$ & 4,63 & $-3,88$ & 1,34 & $-6,39$ & 3,76 & $-3,00$ \\
\hline FEB & 5,92 & $-2,68$ & $-0,46$ & $-7,54$ & 5,21 & $-5,31$ & 2,74 & $-3,86$ \\
\hline MAR & 4,19 & $-3,17$ & 5,46 & $-3,06$ & 6,28 & $-3,06$ & 3,22 & $-4,57$ \\
\hline ABR & 8,79 & $-1,51$ & 6,89 & $-1,27$ & 10,96 & $-0,14$ & 5,94 & $-1,37$ \\
\hline MAY & 10,32 & 1,36 & 14,88 & 4,89 & 14,96 & 3,76 & 9,90 & 1,27 \\
\hline JUN & 21,67 & 9,25 & 21,37 & 10,22 & 19,36 & 7,50 & 15,33 & 4,95 \\
\hline
\end{tabular}


Tabla 4. Media mensual de la amplitud térmica diaria del aire en la estación AIRE (2.255 m s.n.m.).

\begin{tabular}{|l|r|r|r|r|}
\hline \multicolumn{5}{|c|}{ AMPLITUD MEDIA DIARIA } \\
\hline MES & $\mathbf{2 0 0 3 - 0 4}$ & $\mathbf{2 0 0 4 - 0 5}$ & $\mathbf{2 0 0 5 - 0 6}$ & $\mathbf{2 0 0 6 - 0 7}$ \\
\hline OCT & & 6,12 & & 6,08 \\
\hline NOV & 6,13 & 7,47 & & 5,03 \\
\hline DIC & 5,04 & 5,25 & 7,50 & 6,49 \\
\hline ENE & 5,77 & 8,51 & 7,73 & 6,76 \\
\hline FEB & 8,60 & 7,08 & 10,52 & 6,60 \\
\hline MAR & 7,36 & 8,52 & 9,35 & 7,78 \\
\hline ABR & 10,31 & 8,16 & 11,09 & 7,31 \\
\hline MAY & 8,96 & 9,98 & 11,21 & 8,63 \\
\hline JUN & 12,42 & 11,16 & 11,86 & 10,38 \\
\hline
\end{tabular}

\subsection{Temperatura del suelo, estación CUMBRE}

Los resultados de la monitorización de la temperatura del suelo y nieve, en la estación CUMBRE (Fig. 5) dejan constancia de la poca importancia del manto nival en esta estación. La nieve nunca cubre más de $20 \mathrm{~cm}$ la superficie donde se localiza la estación y lo hace por breves períodos. La ausencia total de nieve puede superar el mes, aún en pleno invierno. Este hecho queda muy bien reflejado en los valores obtenidos en el sensor situado a $-10 \mathrm{~cm}$ (Tablas 5, 6, 7 y 8). Las medias mensuales fueron negativas en las cuatro temporadas de enero a marzo, y también lo fueron en el mes de diciembre de 2003 y 2004. Sin embargo, los valores son normalmente más atenuados, aunque muy próximos a los de las medias de la temperatura del aire (contrastar Tablas 1 y 5). A diferencia de lo que ocurre en la temperatura del aire, en los meses invernales las temperaturas máximas diarias permanecen por debajo de $0^{\circ} \mathrm{C}$ en casi todos los casos observados. Así, en el mes de enero se contabilizaron 19, 31, 31 y 31 días con temperaturas negativas constantes en las cuatro campañas. Sin embargo, las diferencias entre las mínimas y las máximas absolutas son muy pequeñas durante los meses invernales, aunque la nieve no esté presente, lo que contrasta de forma considerable con los datos de la temperatura del aire. A la vez que los días de helada se van reduciendo en primavera, la amplitud térmica diaria se va incrementando y supera $1^{\circ} \mathrm{C}$. 


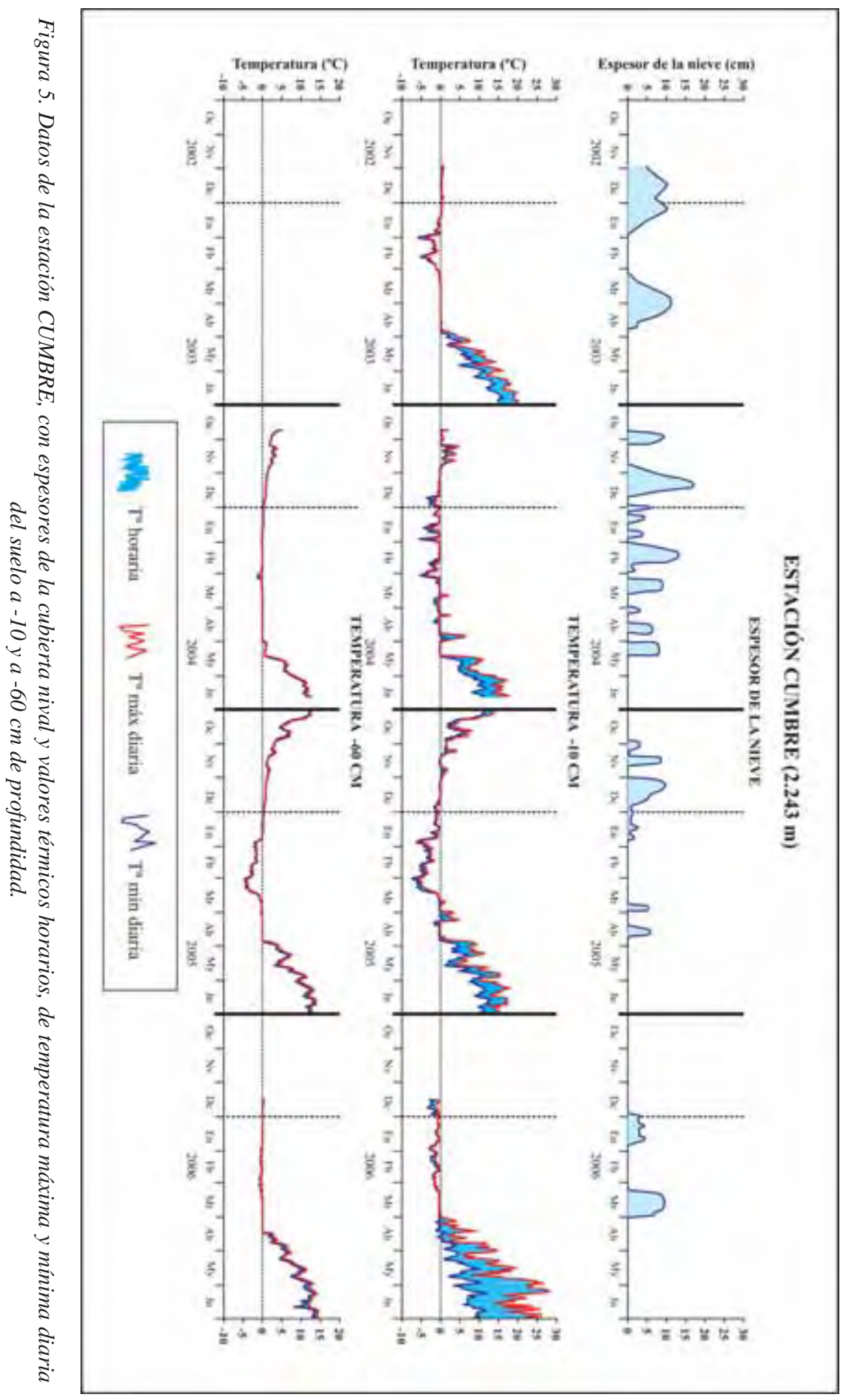


Tabla 5. Temperaturas medias mensuales del suelo a $-10 \mathrm{~cm}$ de la estación CUMBRE $(2.243 \mathrm{~m}$ s.n.m.). (*) Faltan los dos últimos días de junio de 2003. (**) Sólo se registran los primeros 19 días de junio de 2004. (***) Faltan los 15 primeros días de diciembre de 2005.

\begin{tabular}{|l|r|r|r|r|}
\hline \multicolumn{5}{|c|}{$\begin{array}{c}\text { TEMPERATURA MEDIA MENSUAL: } \\
\text { ESTACIÓN CUMBRE - 10 CM }\end{array}$} \\
\hline MES & $\mathbf{2 0 0 2 - 0 3}$ & $\mathbf{2 0 0 3 - 0 4}$ & $\mathbf{2 0 0 4 - 0 5}$ & $\mathbf{2 0 0 5 - 0 6}$ \\
\hline OCT & & & 5,87 & \\
\hline NOV & & 1,10 & 0,69 & \\
\hline DIC & 0,38 & $-0,80$ & $-0,35$ & $-1,45\left(^{* * *}\right)$ \\
\hline ENE & $-0,53$ & $-1,24$ & $-1,84$ & $-1,03$ \\
\hline FEB & $-2,28$ & $-0,99$ & $-3,66$ & $-1,38$ \\
\hline MAR & $-0,21$ & $-0,86$ & $-2,31$ & $-0,54$ \\
\hline ABR & 0,30 & 0,08 & 0,99 & 2,62 \\
\hline MAY & 7,60 & 3,86 & 7,40 & 9,28 \\
\hline JUN & $14,92(*)$ & $12,46(* *)$ & 12,78 & 13,59 \\
\hline
\end{tabular}

Tabla 6. Temperaturas máximas y mínimas absolutas mensuales registradas en el suelo, a $-10 \mathrm{~cm}$, en la estación CUMBRE (2.243 m s.n.m.).

\begin{tabular}{|c|c|c|c|c|c|c|c|c|}
\hline \multicolumn{9}{|c|}{ TEMPERATURA ABSOLUTA HORARIA: ESTACIÓN CUMBRE - 10 CM } \\
\hline \multirow{2}{*}{ MES } & \multicolumn{2}{|c|}{ 2002-03 } & \multicolumn{2}{|c|}{ 2003-04 } & \multicolumn{2}{|c|}{ 2004-05 } & \multicolumn{2}{|c|}{$2005-06$} \\
\hline & $M A X$ & MIN & MAX & MIN & MAX & MIN & MAX & MIN \\
\hline OCT & & & & & 13,88 & 1,02 & & \\
\hline NOV & & & 4,66 & $-0,31$ & 4,20 & $-0,31$ & & \\
\hline DIC & 0,80 & $-0,08$ & $-0,08$ & $-3,64$ & 0,36 & $-1,64$ & $-0,08$ & $-3,21$ \\
\hline ENE & 0,36 & $-5,63$ & $-0,31$ & $-5,63$ & $-0,31$ & $-6,30$ & $-0,08$ & $-2,98$ \\
\hline FEB & $-0,75$ & $-5,63$ & 0,13 & $-3,87$ & $-1,64$ & $-6,30$ & $-0,08$ & $-2,53$ \\
\hline MAR & $-0,08$ & $-1,20$ & 2,15 & $-5,41$ & 3,29 & $-7,42$ & $-0,08$ & $-1,87$ \\
\hline ABR & 3,97 & $-0,08$ & 6,28 & $-1,87$ & 9,13 & $-1,87$ & 14,66 & $-1,20$ \\
\hline MAY & 15,99 & 1,47 & 14,14 & $-0,31$ & 15,45 & 1,02 & 26,86 & 1,02 \\
\hline JUN & 20,22 & 8,65 & 17,63 & 8,17 & 17,91 & 7,46 & 28,25 & 3,29 \\
\hline
\end{tabular}

Tabla 7. Media mensual de las temperaturas máxima y mínima diarias registradas en el suelo, a $-10 \mathrm{~cm}$, en la estación CUMBRE (2.243 m s.n.m.).

\begin{tabular}{|c|c|c|c|c|c|c|c|c|}
\hline \multicolumn{9}{|c|}{ TEMPERATURA MEDIA DIARIA: ESTACIÓN CUMBRE - 10 CM } \\
\hline \multirow{2}{*}{ MES } & \multicolumn{2}{|c|}{$2002-03$} & \multicolumn{2}{|c|}{ 2003-04 } & \multicolumn{2}{|c|}{$2004-05$} & \multicolumn{2}{|c|}{$2005-06$} \\
\hline & $M A X$ & MIN & $M A X$ & MIN & $M A X$ & $M I N$ & $M A X$ & $M I N$ \\
\hline OCT & & & & & 6,80 & 5,06 & & \\
\hline NOV & & & 1,69 & 0,68 & 1,10 & 0,42 & & \\
\hline DIC & 0,44 & 0,33 & $-0,55$ & $-1,03$ & $-0,26$ & $-0,43$ & $-0,81$ & $-2,08$ \\
\hline ENE & $-0,36$ & $-0,70$ & $-0,82$ & $-1,66$ & $-1,51$ & $-2,13$ & $-0,89$ & $-1,15$ \\
\hline FEB & $-1,91$ & $-2,54$ & $-0,72$ & $-1,24$ & $-3,11$ & $-4,06$ & $-1,09$ & $-1,66$ \\
\hline MAR & $-0,19$ & $-0,24$ & $-0,52$ & $-1,14$ & $-1,80$ & $-2,66$ & $-0,47$ & $-0,59$ \\
\hline ABR & 0,54 & 0,13 & 0,83 & $-0,39$ & 2,14 & 0,14 & 5,63 & 0,57 \\
\hline MAY & 9,68 & 5,83 & 5,32 & 2,71 & 9,56 & 5,51 & 15,12 & 5,39 \\
\hline JUN & 17,03 & 12,96 & 15,29 & 9,98 & 15,33 & 10,61 & 21,84 & 8,28 \\
\hline
\end{tabular}


Tabla 8. Media mensual de la amplitud térmica diaria del suelo, a $-10 \mathrm{~cm}$, en la estación CUMBRE (2.243 m s.n.m.).

\begin{tabular}{|l|c|r|r|r|}
\hline \multicolumn{5}{|c|}{ AMPLITUD DIARIA MEDIA: ESTACIÓN CUMBRE - 10 CM } \\
\hline MES & $\mathbf{2 0 0 2 - 0 3}$ & $\mathbf{2 0 0 3 - 0 4}$ & $\mathbf{2 0 0 4 - 0 5}$ & $\mathbf{2 0 0 5 - 0 6}$ \\
\hline OCT & & & 1,74 & \\
\hline NOV & & 1,01 & 0,68 & \\
\hline DIC & 0,11 & 0,47 & 0,17 & 1,27 \\
\hline ENE & 0,34 & 0,85 & 0,62 & 0,27 \\
\hline FEB & 0,63 & 0,52 & 0,96 & 0,57 \\
\hline MAR & 0,05 & 0,62 & 0,85 & 0,12 \\
\hline ABR & 0,40 & 1,22 & 2,00 & 5,06 \\
\hline MAY & 3,84 & 2,61 & 4,05 & 9,72 \\
\hline JUN & 4,07 & 5,31 & 4,72 & 13,56 \\
\hline
\end{tabular}

Si contrastamos estos datos con los datos de la temperatura del suelo en la misma estación CUMBRE a -60 cm (Fig. 5, Tablas 9, 10, 11 y 12), vemos que las medias mensuales están mucho más atenuadas, aunque el frío se mantiene más tiempo en el suelo, por lo que el mes de abril es algo más frío. En las tres campañas se dan medias mensuales negativas en febrero y marzo, dos en enero y una en abril. El número mayor de días de helada al mes se da también entre enero y abril, aunque en enero se registran menos días (12-13-10 respectivamente). Las mínimas absolutas no descienden por debajo de $-1{ }^{\circ} \mathrm{C}$ en la temporada $2005-06$, apenas lo rebasan en $2003-04\left(-1,20^{\circ} \mathrm{C}\right) \mathrm{y}$, de forma anómala, alcanzan $-4,53^{\circ} \mathrm{C}$ en marzo de 2004 . De hecho, la intensidad de la helada disminuye con la profundidad, pero el suelo permanece constantemente helado a esta profundidad los meses de febrero y marzo. Lo más característico de este sensor es que la amplitud térmica es mínima y sólo supera el medio grado a partir de la primavera.

Tabla 9. Temperaturas medias mensuales del suelo a $-60 \mathrm{~cm}$ de la estación CUMBRE $(2.243 \mathrm{~m}$ s.n.m.). (*) Sólo se contabilizan los primeros 19 días de junio de 2004. (**) Faltan los 15 primeros días de diciembre de 2005.

\begin{tabular}{|l|r|r|r|}
\hline \multicolumn{5}{|c|}{$\begin{array}{c}\text { TEMPERATURA MEDIA MENSUAL: } \\
\text { ESTACIÓN CUMBRE - 60 CM }\end{array}$} \\
\hline MES & $\mathbf{2 0 0 3 - 0 4}$ & $\mathbf{2 0 0 4 - 0 5}$ & $\mathbf{2 0 0 5 - 0 6}$ \\
\hline OCT & & 7,45 & \\
\hline NOV & 2,37 & 1,91 & \\
\hline DIC & 0,77 & 0,77 & $0,11\left(^{* *}\right)$ \\
\hline ENE & 0,03 & $-0,26$ & $-0,01$ \\
\hline FEB & $-0,14$ & $-2,17$ & $-0,40$ \\
\hline MAR & $-0,27$ & $-1,92$ & $-0,23$ \\
\hline ABR & $-0,08$ & 0,27 & 1,99 \\
\hline MAY & 3,49 & 6,62 & 8,52 \\
\hline JUN & $10,84(*)$ & 12,07 & 12,56 \\
\hline
\end{tabular}


Tabla 10. Temperaturas máximas y mínimas absolutas mensuales registradas en el suelo, a -60 cm, en la estación CUMBRE (2.243 m s.n.m.).

\begin{tabular}{|l|r|r|r|r|r|r|}
\hline \multicolumn{7}{|c|}{ TEMPERATURA ABSOLUTA HORARIA: ESTACIÓN CUMBRE - 60 CM } \\
\hline \multirow{2}{*}{ MES } & \multicolumn{2}{|c|}{$\mathbf{2 0 0 3 - 0 4}$} & \multicolumn{2}{|c|}{$\mathbf{2 0 0 4 - 0 5}$} & \multicolumn{2}{|c|}{$\mathbf{2 0 0 5 - 0 6}$} \\
\cline { 2 - 7 } & $\boldsymbol{M} \boldsymbol{A} \boldsymbol{X}$ & \multicolumn{1}{c|}{$\boldsymbol{M} \boldsymbol{N}$} & \multicolumn{1}{c|}{$\boldsymbol{M} \boldsymbol{X}$} & \multicolumn{1}{c|}{$\boldsymbol{M I N}$} & \multicolumn{1}{c|}{$\boldsymbol{M} \boldsymbol{X}$} & \multicolumn{1}{c|}{$\boldsymbol{M I N}$} \\
\hline OCT & & & 12,60 & 3,06 & & \\
\hline NOV & 3,74 & 1,25 & 3,52 & 1,02 & & \\
\hline DIC & 1,25 & 0,13 & 1,25 & 0,36 & 0,36 & $-0,08$ \\
\hline ENE & 0,13 & $-0,31$ & 0,36 & $-2,09$ & 0,13 & $-0,53$ \\
\hline FEB & $-0,08$ & $-0,53$ & $-1,20$ & $-3,64$ & $-0,08$ & $-0,75$ \\
\hline MAR & $-0,08$ & $-1,20$ & $-0,08$ & $-4,53$ & $-0,08$ & $-0,75$ \\
\hline ABR & 0,36 & $-0,08$ & 4,20 & $-0,31$ & 6,74 & $-0,08$ \\
\hline MAY & 7,93 & 0,36 & 11,59 & 3,06 & 13,11 & 4,43 \\
\hline JUN & 12,60 & 8,17 & 13,88 & 8,65 & 14,66 & 8,17 \\
\hline
\end{tabular}

Tabla 11. Media mensual de las temperaturas máxima y mínima diarias registradas en el suelo, a $-60 \mathrm{~cm}$, en la estación CUMBRE (2.243 m s.n.m.).

\begin{tabular}{|l|r|r|r|r|r|r|}
\hline \multicolumn{7}{|c|}{$\begin{array}{c}\text { TEMPERATURA MEDIA DIARIA: } \\
\text { ESTACIÓN CUMBRE - 60 CM }\end{array}$} \\
\hline \multirow{2}{*}{ MES } & \multicolumn{2}{|c|}{$\mathbf{2 0 0 3 - 0 4}$} & \multicolumn{2}{c|}{$\mathbf{2 0 0 4 - 0 5}$} & \multicolumn{2}{c|}{$\mathbf{2 0 0 5 - 0 6}$} \\
\cline { 2 - 8 } & $\boldsymbol{M} \boldsymbol{X}$ & $\boldsymbol{M I N}$ & $\boldsymbol{M} \boldsymbol{A} \boldsymbol{X}$ & \multicolumn{1}{c|}{$\boldsymbol{M I N}$} & \multicolumn{1}{c|}{$\boldsymbol{M} \boldsymbol{A} \boldsymbol{X}$} & \multicolumn{1}{c|}{$\boldsymbol{M I N}$} \\
\hline OCT & & & 7,77 & 7,15 & & \\
\hline NOV & 2,49 & 2,24 & 2,07 & 1,80 & & 0,08 \\
\hline DIC & 0,80 & 0,75 & 0,79 & 0,75 & 0,15 & $-0,04$ \\
\hline ENE & 0,03 & 0,01 & $-0,19$ & $-0,32$ & 0,04 & $-0,44$ \\
\hline FEB & $-0,12$ & $-0,17$ & $-1,99$ & $-2,29$ & $-0,37$ & $-0,25$ \\
\hline MAR & $-0,21$ & $-0,29$ & $-1,78$ & $-2,04$ & $-0,21$ & 1,74 \\
\hline ABR & $-0,07$ & $-0,08$ & 0,37 & 0,20 & 2,33 & 8,06 \\
\hline MAY & 3,68 & 3,33 & 7,13 & 6,25 & 9,17 & 11,94 \\
\hline JUN & 11,12 & 10,53 & 12,55 & 11,64 & 13,19 & \\
\hline
\end{tabular}

Tabla 12. Media mensual de la amplitud térmica diaria del suelo, a $-60 \mathrm{~cm}$, en la estación CUMBRE (2.243 m s.n.m.).

\begin{tabular}{|l|r|r|r|}
\hline \multicolumn{4}{|c|}{ AMPLITUD DIARIA MEDIA: ESTACIÓN } \\
CUMBRE - 60 CM \\
\hline & $\mathbf{2 0 0 3 - 0 4}$ & $\mathbf{2 0 0 4 - 0 5}$ & $\mathbf{2 0 0 5 - 0 6}$ \\
\hline OCT & & 0,62 & \\
\hline NOV & 0,25 & 0,27 & \\
\hline DIC & 0,05 & 0,04 & 0,07 \\
\hline ENE & 0,02 & 0,13 & 0,08 \\
\hline FEB & 0,06 & 0,30 & 0,06 \\
\hline MAR & 0,08 & 0,26 & 0,04 \\
\hline ABR & 0,01 & 0,17 & 0,59 \\
\hline MAY & 0,35 & 0,88 & 1,11 \\
\hline JUN & 0,59 & 0,91 & 1,26 \\
\hline
\end{tabular}




\subsection{Temperatura del suelo, estación NIVAL}

Los resultados del seguimiento de la temperatura del suelo, en la estación NIVAL (Fig. 6) muestran cómo la nieve permanece de forma constante y con un gran espesor durante toda la temporada. El espesor de la nieve supera ya $\operatorname{los} 20 \mathrm{~cm}$ a finales del otoño $\mathrm{y}$ desde entonces hasta finales de mayo o mediados de junio permanece constante sobre esta estación. Una amplitud térmica diaria nula o próxima a $0^{\circ} \mathrm{C}$ es el indicio de la existencia de una capa de nieve capaz de aislar el suelo de la influencia de las oscilaciones de la temperatura del aire.

En el sensor de -10 cm de esta estación NIVAL (Tablas 13, 14, 15 y 16) refleja la influencia de la nieve en el régimen térmico del suelo. La mayor parte de las medias mensuales y las máximas y mínimas absolutas se encuentran alrededor de los $0^{\circ} \mathrm{C}$, tendiendo a que las décimas sean positivas. Durante el inicio de la temporada de presencia de nieve en el suelo se observa que las amplitudes diarias se van reduciendo paulatinamente, aunque su descenso se ve interrumpido por la influencia del incremento en la temperatura del aire. La protección del suelo se puede iniciar desde finales de octubre a principios de diciembre. En cambio, en primavera el cese del aislamiento térmico se produce de forma brusca entre finales de mayo y finales de junio. Por ejemplo, en diciembre de 2002 la oscilación ya era mínima $\left(0,01^{\circ} \mathrm{C}\right)$, aunque no se tienen registros anteriores; en noviembre de $2003,0,1^{\circ} \mathrm{C}$; en diciembre de $2004,0,01^{\circ} \mathrm{C}$ y en diciembre de $200,0,16^{\circ} \mathrm{C}$. Únicamente cuando la cubierta nival supera los $20 \mathrm{~cm}$, normalmente a mediados de diciembre, la oscilación térmica es nula o mínima (inferior a los $0,1^{\circ} \mathrm{C}$ ). En la primavera la oscilación térmica se dispara el día que la nieve desaparece. Por ejemplo, entre el 19 y el 20 de junio de 2003 la amplitud pasa de 0 a $9,21^{\circ} \mathrm{C}$; entre el 27 y el 28 de junio de 2004 la oscilación diaria de la temperatura pasa de $0,21^{\circ} \mathrm{C}$ a $10,96^{\circ} \mathrm{C}$; entre el 1 y el 2 de junio en 2005 pasa de 0,44 a $6,4^{\circ} \mathrm{C}$; entre el 30 y el 31 de mayo de 2006 pasa de 0 a $14,74^{\circ} \mathrm{C}$.

Tabla 13. Temperaturas medias mensuales del suelo a $-10 \mathrm{~cm}$ de la estación NIVAL $(2.212 \mathrm{~m}$ s.n.m.) (*) Faltan los dos últimos días de junio de 2003. (**) Faltan los

14 primeros días de diciembre de 2005.

\begin{tabular}{|l|r|r|r|r|}
\hline \multicolumn{7}{|c|}{$\begin{array}{c}\text { TEMPERATURA MEDIA MENSUAL: } \\
\text { ESTACIÓN NIVAL-10 CM }\end{array}$} \\
\hline MES & $\mathbf{2 0 0 2 - 0 3}$ & $\mathbf{2 0 0 3 - 0 4}$ & $\mathbf{2 0 0 4 - 0 5}$ & $\mathbf{2 0 0 5 - 0 6}$ \\
\hline OCT & & & 6,26 & \\
\hline NOV & & 0,43 & 0,70 & \\
\hline DIC & 0,25 & 0,36 & 0,29 & $-0,02\left(^{* *}\right)$ \\
\hline ENE & 0,13 & 0,36 & 0,13 & 0,10 \\
\hline FEB & 0,13 & 0,36 & 0,13 & $-0,03$ \\
\hline MAR & 0,13 & 0,27 & 0,13 & $-0,08$ \\
\hline ABR & 0,12 & 0,13 & 0,12 & $-0,08$ \\
\hline MAY & $-0,08$ & 0,10 & $-0,08$ & 0,07 \\
\hline JUN & $3,92(*)$ & 0,85 & 11,52 & 11,10 \\
\hline
\end{tabular}


Tabla 14. Temperaturas máximas y mínimas absolutas mensuales registradas en el suelo, a $-10 \mathrm{~cm}$, en la estación NIVAL (2.212 m s.n.m.).

\begin{tabular}{|c|c|c|c|c|c|c|c|c|}
\hline \multicolumn{9}{|c|}{ TEMPERATURA ABSOLUTA HORARIA: ESTACIÓN NIVAL - 10 CM } \\
\hline \multirow{2}{*}{ MES } & \multicolumn{2}{|c|}{ 2002-03 } & \multicolumn{2}{|c|}{ 2003-04 } & \multicolumn{2}{|c|}{ 2004-05 } & \multicolumn{2}{|c|}{ 2005-06 } \\
\hline & $M A X$ & MIN & $\boldsymbol{M A X}$ & MIN & $\boldsymbol{M A X}$ & MIN & $M A X$ & $M I N$ \\
\hline OCT & & & & & 16,26 & 1,25 & & \\
\hline NOV & & & 0,80 & 0,13 & 3,29 & 0,13 & & \\
\hline DIC & 0,58 & $-0,08$ & 0,36 & 0,36 & 0,58 & 0,13 & 0,13 & $-0,08$ \\
\hline ENE & 0,13 & 0,13 & 0,36 & 0,36 & 0,13 & 0,13 & 0,13 & $-0,08$ \\
\hline FEB & 0,13 & 0,13 & 0,36 & 0,36 & 0,13 & 0,13 & 0,13 & $-0,08$ \\
\hline MAR & 0,13 & 0,13 & 0,36 & 0,13 & 0,13 & 0,13 & $-0,08$ & $-0,08$ \\
\hline ABR & 0,13 & $-0,08$ & 0,13 & 0,13 & 0,13 & $-0,08$ & $-0,08$ & $-0,08$ \\
\hline MAY & $-0,08$ & $-0,08$ & 0,13 & $-0,08$ & $-0,08$ & $-0,08$ & 14,66 & $-0,08$ \\
\hline JUN & 20,51 & $-0,08$ & 18,48 & $-0,08$ & 17,36 & $-0,08$ & 24,85 & 0,13 \\
\hline
\end{tabular}

Tabla 15. Media mensual de las temperaturas máximas y mínimas diarias registradas en el suelo, a $-10 \mathrm{~cm}$, en la estación NIVAL (2.212 m s.n.m.).

\begin{tabular}{|c|c|c|c|c|c|c|c|c|}
\hline \multicolumn{9}{|c|}{ TEMPERATURA MEDIA DIARIA: ESTACIÓN NIVAL - 10 CM } \\
\hline & \multicolumn{2}{|c|}{ 2002-03 } & \multicolumn{2}{|c|}{ 2003-04 } & \multicolumn{2}{|c|}{ 2004-05 } & \multicolumn{2}{|c|}{ 2005-06 } \\
\hline & $M A X$ & $M I N$ & $M A X$ & MIN & $M A X$ & MIN & MAX & MIN \\
\hline OCT & & & & & 7,42 & 5,22 & & \\
\hline NOV & & & 0,48 & 0,38 & 0,95 & 0,51 & & \\
\hline DIC & 0,26 & 0,21 & 0,36 & 0,36 & 0,30 & 0,29 & 0,08 & $-0,08$ \\
\hline ENE & 0,13 & 0,13 & 0,36 & 0,36 & 0,13 & 0,13 & 0,13 & 0,07 \\
\hline FEB & 0,13 & 0,13 & 0,36 & 0,36 & 0,13 & 0,13 & 0,05 & $-0,08$ \\
\hline MAR & 0,13 & 0,13 & 0,29 & 0,23 & 0,13 & 0,13 & $-0,08$ & $-0,08$ \\
\hline ABR & 0,12 & 0,11 & 0,13 & 0,13 & 0,12 & 0,12 & $-0,08$ & $-0,08$ \\
\hline MAY & $-0,08$ & $-0,08$ & 0,12 & 0,08 & $-0,08$ & $-0,08$ & 0,43 & $-0,08$ \\
\hline JUN & 5,31 & 2,72 & 1,49 & 0,40 & 14,09 & 9,16 & 17,92 & 5,85 \\
\hline
\end{tabular}

Tabla 16. Media mensual de la amplitud térmica diaria del suelo, a $-10 \mathrm{~cm}$, en la estación NIVAL (2.212 m s.n.m.).

\begin{tabular}{|l|c|r|r|r|}
\hline \multicolumn{5}{|c|}{ AMPLITUD DIARIA MEDIA: ESTACIÓN NIVAL - 10 CM } \\
\hline & $\mathbf{2 0 0 2 - 0 3}$ & $\mathbf{2 0 0 3 - 0 4}$ & $\mathbf{2 0 0 4 - 0 5}$ & $\mathbf{2 0 0 5 - 0 6}$ \\
\hline OCT & & & 2,20 & \\
\hline NOV & & 0,10 & 0,43 & \\
\hline DIC & 0,05 & 0,00 & 0,01 & 0,16 \\
\hline ENE & 0,00 & 0,00 & 0,00 & 0,06 \\
\hline FEB & 0,00 & 0,00 & 0,00 & 0,13 \\
\hline MAR & 0,00 & 0,06 & 0,00 & 0,00 \\
\hline ABR & 0,01 & 0,00 & 0,01 & 0,00 \\
\hline MAY & 0,00 & 0,04 & 0,00 & 0,51 \\
\hline JUN & 2,59 & 1,09 & 4,93 & 12,06 \\
\hline
\end{tabular}




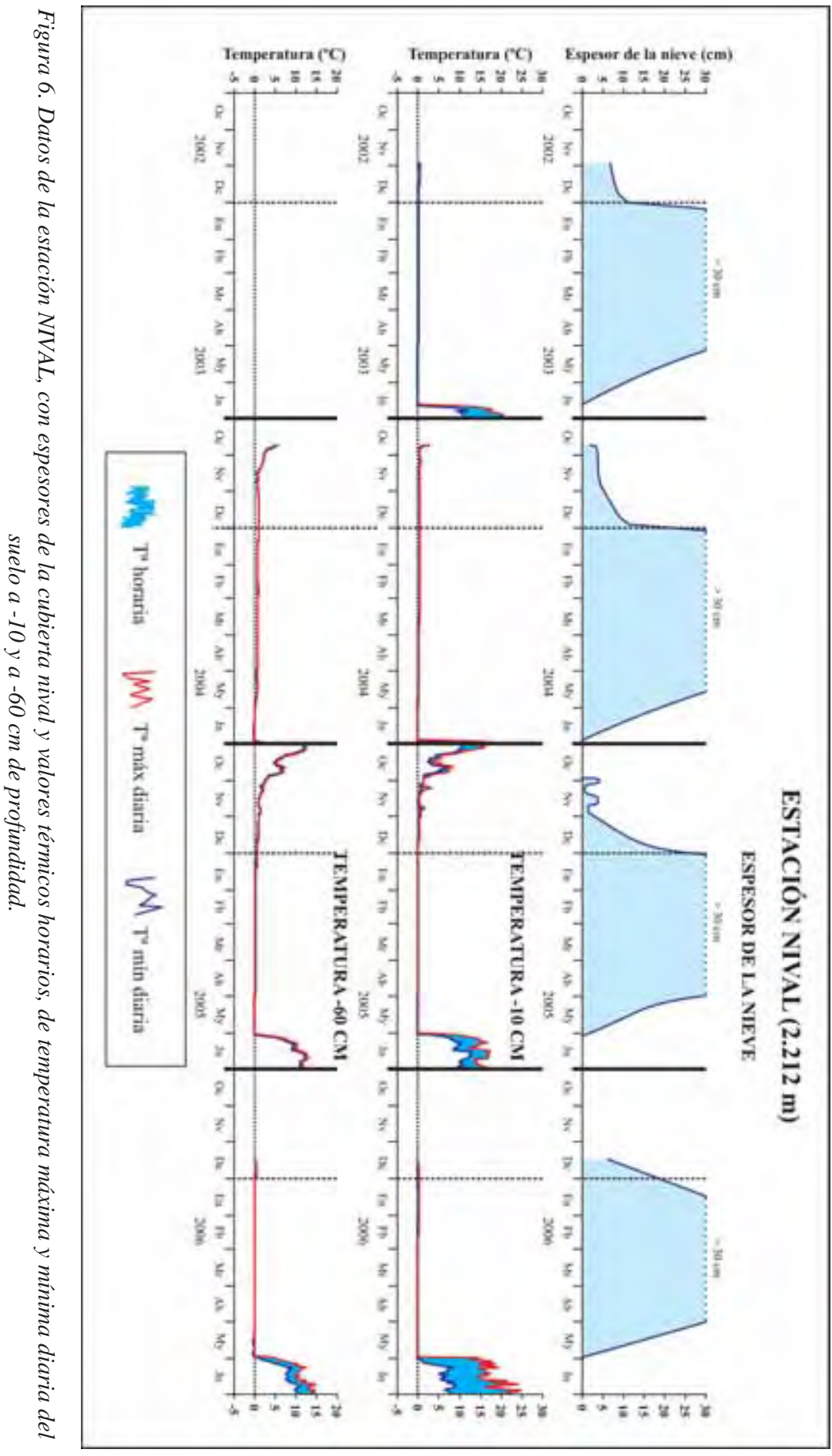


Mientras dura la cubierta nival las temperaturas medias mensuales se mantienen próximas a $0^{\circ} \mathrm{C}$, pero positivas, excepto en los meses de mayo de 2003 y 2005, en los que todos los días se registraron temperaturas máximas absolutas negativas de $-0,08^{\circ} \mathrm{C}$, aunque lo suficientemente cercanas a $0^{\circ} \mathrm{C}$ para que el agua se mantenga en estado líquido (situación criótica). En mayo de 2004 también se anotaron máximas diarias negativas ( 2 días) y oscilaciones diarias alrededor de $0^{\circ} \mathrm{C}$ ( 6 días con máxima positiva y mínima negativa). Según esto, se constata que las temperaturas van descendiendo, muy poco a poco a lo largo del invierno y principios de la primavera y las temperaturas más bajas se producen al final de la primavera, cerca del momento de fusión de la nieve.

Sin embargo, la campaña 2005-06 no sigue este esquema, ya que los meses de febrero, marzo y abril tuvieron medias mensuales inferiores a $0^{\circ} \mathrm{C}$. Entre febrero y mayo todos los días registraron mínimas negativas y sólo 17 días de febrero y 2 en mayo tuvieron máximas positivas. Las temperaturas del aire observadas durante el invierno 2005-06 fueron mucho más bajas que las de los años anteriores (enero de 2006 tiene una media de $-5,02^{\circ} \mathrm{C}$, mientras que en 2004 tuvo $-0,70^{\circ} \mathrm{C}$ y en $2005,-0,80^{\circ} \mathrm{C}$ ). El mes de febrero fue algo más cálido que el de 2005, pero de nuevo en marzo volvieron a caer las temperaturas con valores inferiores a los de años anteriores. Abril y mayo presentan medias del aire similares a las registradas en 2005, pero la acumulación del frío en el suelo mantuvo las temperaturas del suelo más frías en 2006 que en 2005.

Los resultados de la monitorización de la temperatura del suelo del sensor situado a $-60 \mathrm{~cm}$ de profundidad, en la estación NIVAL (Fig. 6, Tablas 17, 18, 19 y 20) presenta datos, tanto de las medias diarias, mensuales y absolutas, muy similares a las de la superficie, siempre con valores muy próximos a los $0^{\circ} \mathrm{C}$. La diferencia es que el enfriamiento del suelo se va haciendo con retraso, con respecto al sensor de $-10 \mathrm{~cm}$ y las oscilaciones son aún menores. Por ejemplo, el 1 de noviembre de 2003 la media en superficie es de $0,58^{\circ} \mathrm{C}$ mientras que en profundidad se registran $2,15^{\circ} \mathrm{C}$. En profundidad no se dan temperaturas medias diarias inferiores a $1^{\circ} \mathrm{C}$ hasta el 15 de

Tabla 17. Temperaturas medias mensuales del suelo a $-60 \mathrm{~cm}$ de la estación NIVAL (2.212 m s.n.m.). (*) Faltan los 14 primeros días de diciembre de 2005.

\begin{tabular}{|l|c|r|r|}
\hline \multicolumn{4}{|c|}{$\begin{array}{c}\text { TEMPERATURA MEDIA MENSUAL: } \\
\text { ESTACIÓN NIVAL - 60 CM }\end{array}$} \\
\hline MES & $\mathbf{2 0 0 3 - 0 4}$ & $\mathbf{2 0 0 4 - 0 5}$ & $\mathbf{2 0 0 5 - 0 6}$ \\
\hline OCT & & 7,20 & \\
\hline NOV & 1,26 & 1,42 & $0,28\left(^{*}\right)$ \\
\hline DIC & 1,00 & 0,74 & 0,13 \\
\hline ENE & 0,88 & 0,42 & 0,13 \\
\hline FEB & 0,81 & 0,36 & 0,13 \\
\hline MAR & 0,80 & 0,36 & $-0,08$ \\
\hline ABR & 0,79 & 0,26 & 0,03 \\
\hline MAY & 0,51 & $-0,08$ & 9,60 \\
\hline JUN & 0,02 & 9,35 & \\
\hline
\end{tabular}


Tabla 18. Temperaturas máximas y mínimas absolutas mensuales registradas en el suelo, a -60 cm, en la estación NIVAL (2.212 m s.n.m.).

\begin{tabular}{|c|c|c|c|c|c|c|}
\hline \multicolumn{7}{|c|}{ TEMPERATURA ABSOLUTA HORARIA: ESTACIÓN NIVAL - 60 CM } \\
\hline \multirow{2}{*}{ MES } & \multicolumn{2}{|c|}{ 2003-04 } & \multicolumn{2}{|c|}{$2004-05$} & \multicolumn{2}{|c|}{$2005-06$} \\
\hline & $M A X$ & MIN & $M A X$ & MIN & $M A X$ & $M I N$ \\
\hline OCT & & & 12,34 & 2,61 & & \\
\hline $\mathrm{NOV}$ & 2,15 & 0,58 & 2,61 & 0,80 & & \\
\hline DIC & 1,02 & 0,80 & 1,02 & 0,58 & 0,36 & 0,13 \\
\hline ENE & 1,02 & 0,80 & 0,58 & 0,36 & 0,13 & 0,13 \\
\hline FEB & 1,02 & 0,80 & 0,36 & 0,36 & 0,13 & 0,13 \\
\hline MAR & 0,80 & 0,80 & 0,36 & 0,13 & 0,13 & $-0,08$ \\
\hline $\mathrm{ABR}$ & 0,80 & 0,58 & 0,36 & $-0,08$ & 0,13 & $-0,08$ \\
\hline MAY & 0,80 & $-0,08$ & 0,13 & $-0,08$ & 5,12 & $-0,31$ \\
\hline JUN & 3,97 & $-0,31$ & 12,86 & $-0,08$ & 14,66 & 1,47 \\
\hline
\end{tabular}

Tabla 19. Media mensual de las temperaturas máxima y mínima diarias registradas en el suelo, a -60 cm, en la estación NIVAL (2.212 m s.n.m.).

\begin{tabular}{|c|c|c|c|c|c|c|}
\hline \multicolumn{7}{|c|}{ TEMPERATURA MEDIA DIARIA: ESTACIÓN NIVAL - 60 CM } \\
\hline \multirow{2}{*}{ MES } & \multicolumn{2}{|c|}{ 2003-04 } & \multicolumn{2}{|c|}{ 2004-05 } & \multicolumn{2}{|c|}{$2005-06$} \\
\hline & $M A X$ & $M I N$ & $M A X$ & $M I N$ & $M A X$ & $M I N$ \\
\hline OCT & & & 7,56 & 6,89 & & \\
\hline NOV & 1,32 & 1,20 & 1,54 & 1,34 & & \\
\hline DIC & 1,01 & 1,00 & 0,76 & 0,73 & 0,32 & 0,24 \\
\hline ENE & 0,89 & 0,87 & 0,45 & 0,40 & 0,13 & 0,13 \\
\hline FEB & 0,88 & 0,80 & 0,36 & 0,36 & 0,13 & 0,13 \\
\hline MAR & 0,80 & 0,80 & 0,36 & 0,35 & 0,13 & 0,12 \\
\hline ABR & 0,79 & 0,79 & 0,28 & 0,23 & $-0,07$ & $-0,08$ \\
\hline MAY & 0,53 & 0,49 & $-0,07$ & $-0,08$ & 0,17 & $-0,14$ \\
\hline JUN & 0,12 & $-0,10$ & 9,89 & 8,96 & 11,33 & 7,89 \\
\hline
\end{tabular}

Tabla 20. Media mensual de la amplitud térmica diaria del suelo, a $-60 \mathrm{~cm}$, en la estación NIVAL (2.212 m s.n.m.).

\begin{tabular}{|l|c|r|r|}
\hline \multicolumn{4}{|c|}{ AMPLITUD DIARIA MEDIA: ESTACIÓN } \\
NIVAL- 60 CM \\
\hline MES & $\mathbf{2 0 0 3 - 0 4}$ & $\mathbf{2 0 0 4 - 0 5}$ & $\mathbf{2 0 0 5 - 0 6}$ \\
\hline OCT & & 0,67 & \\
\hline NOV & 0,12 & 0,20 & \\
\hline DIC & 0,01 & 0,04 & 0,08 \\
\hline ENE & 0,01 & 0,06 & 0,00 \\
\hline FEB & 0,08 & 0,00 & 0,00 \\
\hline MAR & 0,00 & 0,01 & 0,01 \\
\hline ABR & 0,01 & 0,05 & 0,01 \\
\hline MAY & 0,04 & 0,01 & 0,31 \\
\hline JUN & 0,22 & 0,93 & 3,43 \\
\hline
\end{tabular}


noviembre, mucho después que a $-10 \mathrm{~cm}$. Lo mismo ocurre en el otoño de 2004 , donde a $-10 \mathrm{~cm}$ se registra la primera media diaria inferior a $1{ }^{\circ} \mathrm{C}$ de forma continuada a partir del 11 de noviembre. A $-60 \mathrm{~cm}$, la primera media diaria inferior a $1{ }^{\circ} \mathrm{C} \mathrm{no}$ se da hasta el 6 de diciembre. En 2005, los últimos 17 días de diciembre tienen medias diarias negativas a $-10 \mathrm{~cm}\left(-0,08^{\circ} \mathrm{C}\right)$, mientras que tienen medias positivas a $-60 \mathrm{~cm}$ $\left(0,13^{\circ} \mathrm{C}\right)$ y no se alcanza una temperatura mínima diaria negativa hasta el 31 de marzo del $2006\left(-0,08^{\circ} \mathrm{C}\right)$. Del mismo modo que ocurría a $-10 \mathrm{~cm}$, pero de forma más marcada, las temperaturas más bajas se registran a finales de la primavera, justo antes de la fusión de la nieve.

\subsection{Temperatura del suelo, estación PRONIVAL}

Los resultados de la monitorización de la temperatura del suelo, en la estación PRONIVAL (Fig. 7) muestran una situación de la nieve intermedia entre la estación CUMBRE y la estación NIVAL. La nieve permanece desde finales de noviembre de forma estable en el suelo, salvo excepciones, aunque con un espesor reducido, en torno a los 20/30 cm. La nieve se retira pronto, normalmente a mediados o finales de abril. Sin embargo, los valores térmicos son bastante semejantes a los de la estación NIVAL, entre los meses de diciembre a marzo.

La sonda de $-10 \mathrm{~cm}$ muestra unas medias mensuales muy próximas a $0^{\circ} \mathrm{C}$ y en la mayor parte de las temporadas positivas (Fig. 7 y Tablas 21, 22, 23 y 24). Las mínimas absolutas son altas, normalmente no llegan a $-1^{\circ} \mathrm{C}$, salvo el excepcional mes de febrero de 2003, cuando la estación no estuvo cubierta por la nieve. Los valores, a partir del mes de abril o mayo, según las temporadas, reflejan ya los cambios térmicos del aire y la amplitud, prácticamente nula desde diciembre a marzo, asciende entonces considerablemente. Por ejemplo, la amplitud es inferior a $0,5^{\circ} \mathrm{C}$ entre diciembre y marzo en la campaña 2002-03; entre noviembre y marzo en la campaña 2003-04; y entre diciembre y febrero en la de 2006-07.

Tabla 21. Temperaturas medias mensuales del suelo a $-10 \mathrm{~cm}$ de la estación PRONIVAL (2.163 m s.n.m.). (*) Faltan dos días de junio de 2003. (**) Sólo se emplean los 19 primeros días de junio de 2004.

\begin{tabular}{|l|r|r|r|}
\hline \multicolumn{5}{|c|}{$\begin{array}{c}\text { TEMPERATURA MEDIA MENSUAL: } \\
\text { ESTACIÓN PRONIVAL - 10 CM }\end{array}$} \\
\hline MES & $\mathbf{2 0 0 2 - 0 3}$ & $\mathbf{2 0 0 3 - 0 4}$ & $\mathbf{2 0 0 6 - 0 7}$ \\
\hline NOV & & 2,43 & 4,93 \\
\hline DIC & 0,15 & 1,02 & 0,59 \\
\hline ENE & $-0,10$ & 0,61 & 0,56 \\
\hline FEB & $-0,59$ & 0,41 & 0,63 \\
\hline MAR & $-0,33$ & 0,30 & 0,72 \\
\hline ABR & 0,36 & 1,69 & 2,25 \\
\hline MAY & 8,31 & 4,64 & 7,03 \\
\hline JUN & $15,02(*)$ & $13,91\left(^{* *}\right)$ & 11,03 \\
\hline
\end{tabular}


Tabla 22. Temperaturas máximas y mínimas absolutas mensuales registradas en el suelo, a -10 cm, en la estación PRONIVAL (2.163 m s.n.m.).

\begin{tabular}{|c|c|c|c|c|c|c|}
\hline \multicolumn{7}{|c|}{ TEMPERATURA ABSOLUTA HORARIA: ESTACIÓN PRO NIVAL - 10 CM } \\
\hline \multirow{2}{*}{ MES } & \multicolumn{2}{|c|}{ 2002-03 } & \multicolumn{2}{|c|}{ 2003-04 } & \multicolumn{2}{|c|}{ 2006-07 } \\
\hline & MAX & MIN & $M A X$ & MIN & $M A X$ & MIN \\
\hline NOV & & & 4,20 & 1,02 & 10,10 & 1,70 \\
\hline DIC & 1,02 & $-0,31$ & 1,25 & 1,02 & 3,29 & $-0,97$ \\
\hline ENE & 1,47 & $-0,31$ & 1,02 & 0,13 & 3,97 & $-0,31$ \\
\hline FEB & $-0,31$ & $-1,87$ & 0,58 & 0,13 & 0,80 & 0,36 \\
\hline MAR & 0,13 & $-0,53$ & 0,58 & 0,13 & 4,43 & 0,13 \\
\hline $\mathrm{ABR}$ & 5,81 & $-0,31$ & 10,59 & 0,13 & 8,41 & 0,36 \\
\hline MAY & 16,26 & 1,47 & 11,59 & 0,58 & 11,33 & 2,38 \\
\hline JUN & 20,22 & 9,13 & 17,08 & 11,59 & 17,08 & 7,22 \\
\hline
\end{tabular}

Tabla 23. Media mensual de las temperaturas máxima y mínima diarias registradas en el suelo, a -10 cm, en la estación PRONIVAL (2.163 m s.n.m.).

\begin{tabular}{|c|c|c|c|c|c|c|}
\hline \multicolumn{7}{|c|}{ TEMPERATURA MEDIA DIARIA: ESTACIÓN PRONIVAL - 10 CM } \\
\hline \multirow{2}{*}{ MES } & \multicolumn{2}{|c|}{ 2002-03 } & \multicolumn{2}{|c|}{ 2003-04 } & \multicolumn{2}{|c|}{ 2006-07 } \\
\hline & MAX & $M I N$ & MAX & $M I N$ & $M A X$ & $M I N$ \\
\hline NOV & & & 2,65 & 2,22 & 5,68 & 4,33 \\
\hline DIC & 0,24 & 0,09 & 1,04 & 1,02 & 0,77 & 0,37 \\
\hline ENE & $-0,02$ & $-0,15$ & 0,63 & 0,57 & 0,74 & 0,44 \\
\hline FEB & $-0,55$ & $-0,64$ & 0,44 & 0,37 & 0,67 & 0,57 \\
\hline MAR & $-0,30$ & $-0,35$ & 0,32 & 0,28 & 1,12 & 0,49 \\
\hline ABR & 0,68 & 0,11 & 2,26 & 1,32 & 2,71 & 1,83 \\
\hline MAY & 10,46 & 6,39 & 5,24 & 4,19 & 8,15 & 6,15 \\
\hline JUN & 17,07 & 13,13 & 15,00 & 12,90 & 13,09 & 9,38 \\
\hline
\end{tabular}

Tabla 24. Media mensual de la amplitud térmica diaria del suelo, a $-10 \mathrm{~cm}$, en la estación PRONIVAL (2.163 m s.n.m.).

\begin{tabular}{|l|c|r|r|}
\hline \multicolumn{4}{|c|}{ AMPLITUD DIARIA MEDIA: } \\
ESTACIÓN PRONIVAL-10 CM \\
\hline & $\mathbf{2 0 0 2 - 0 3}$ & $\mathbf{2 0 0 3 - 0 4}$ & $\mathbf{2 0 0 6 - 0 7}$ \\
\hline NOV & & 0,43 & 1,35 \\
\hline DIC & 0,16 & 0,02 & 0,40 \\
\hline ENE & 0,13 & 0,06 & 0,30 \\
\hline FEB & 0,10 & 0,07 & 0,09 \\
\hline MAR & 0,04 & 0,04 & 0,62 \\
\hline ABR & 0,58 & 0,94 & 0,87 \\
\hline MAY & 4,07 & 1,05 & 2,00 \\
\hline JUN & 3,95 & 2,10 & 3,71 \\
\hline
\end{tabular}




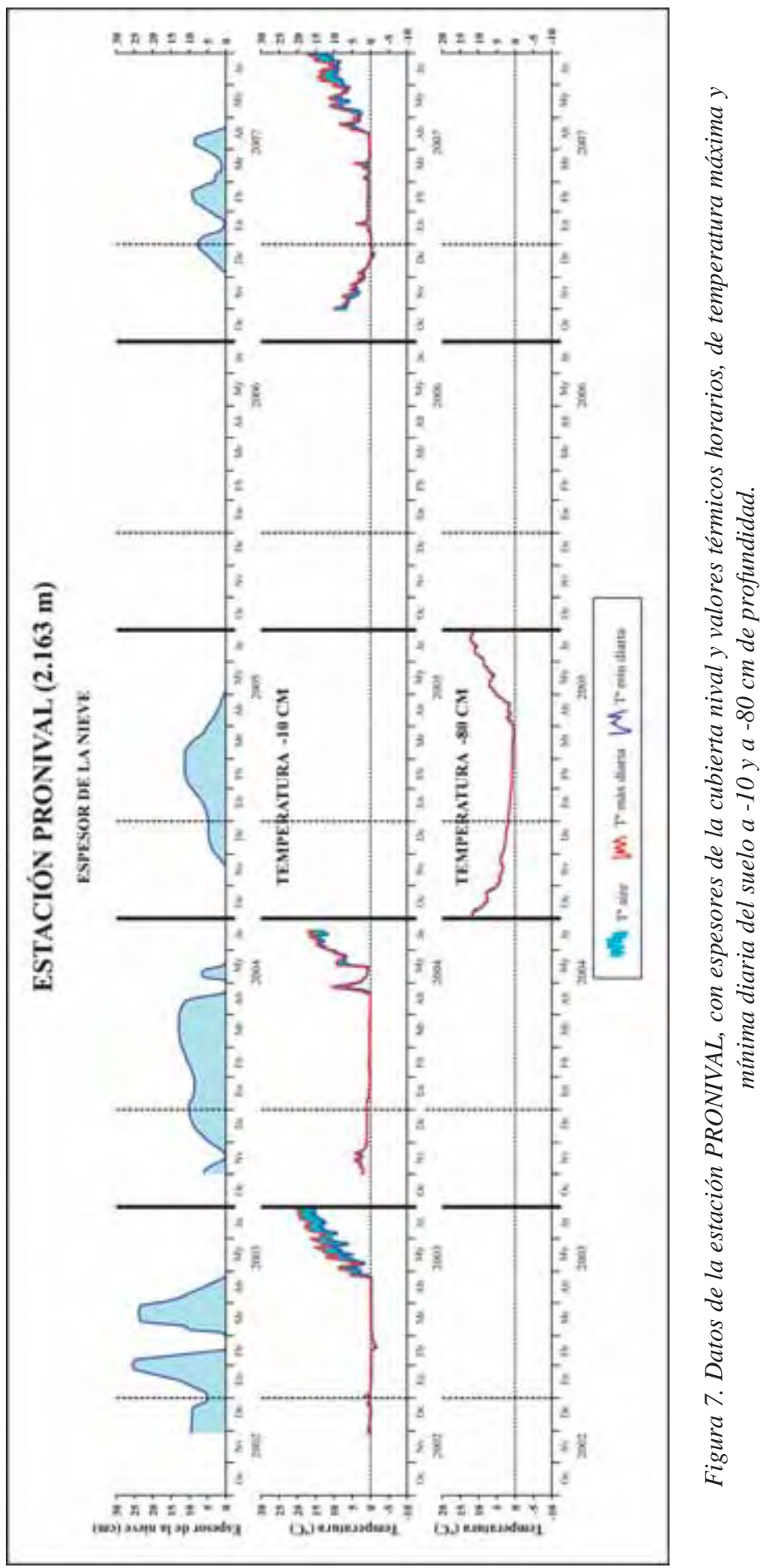


El suelo estuvo helado muy poco tiempo durante todo el período de observación. El único período destacable fue precisamente en la temporada 2002-03, cuando durante parte del mes de enero y todo febrero desapareció el manto nival. Esto permitió la intensa penetración del frío en el suelo, que después, cuando volvió la cubierta nival, quedó aislado en el suelo. Por este motivo, el suelo estuvo por debajo de $\operatorname{los} 0^{\circ} \mathrm{C} 23$ días en enero de 2003, todo el mes de febrero, y gran parte de los meses de marzo (30 días) y abril (23 días). Por otro lado, esto permitió el registro de la mínima absoluta más baja de todo el período de observación, que fue el 18 de febrero, con $-1,87^{\circ} \mathrm{C}$. El suelo no volvió a estar por debajo de los $0^{\circ} \mathrm{C}$ hasta 12 días en diciembre del 2006 y 1 en enero, con mínimas absolutas que alcanzaron $\operatorname{los}-0,97^{\circ} \mathrm{C}(22-12-2006)$, al coincidir también con un breve período de ausencia de nieve.

Durante la única temporada en la que se pudo obtener datos de la sonda de -80 cm (Fig. 7 y Tabla 25) en la estación PRONIVAL, se observó cómo a esa profundidad las temperaturas permanecen siempre por encima de los $0^{\circ} \mathrm{C}$. Sin embargo, las temperaturas van descendiendo poco a poco a lo largo del invierno y las mínimas absolutas se registran en marzo y abril $\left(0,58^{\circ} \mathrm{C}\right)$. La amplitud diaria media mensual es un poco más reducida durante la época nival: de diciembre a marzo varía entre 0,03 y $0,06^{\circ} \mathrm{C}$. Se incrementa cuando la nieve desaparece, pero siempre se mantiene por debajo de $0,30^{\circ} \mathrm{C}$.

Tabla 25. Temperaturas medias mensuales (TM MEN), temperaturas máximas y mínimas absolutas mensuales (T ABS), medias mensuales de las temperaturas máximas y mínimas diarias (T MED DIA) y medias mensuales de la amplitud térmica diaria (A M) del suelo a $-80 \mathrm{~cm}$, en la estación PRONIVAL (2.163 m s.n.m.) durante la temporada 2004-2005.

\begin{tabular}{|l|r|r|r|r|r|r|}
\hline \multicolumn{7}{|c|}{ TEMPERATURA DE LA ESTACIÓN PRONIVAL - 80 CM 2004-05 } \\
\hline \multirow{2}{*}{ MES } & TM MEN & \multicolumn{2}{|c|}{ T ABS } & \multicolumn{1}{c|}{ T MED DIA } & \multicolumn{1}{c|}{ A M } \\
\hline & & \multicolumn{1}{c}{$\boldsymbol{M} \boldsymbol{X}$} & \multicolumn{1}{c|}{$\boldsymbol{M} \boldsymbol{N}$} & $\boldsymbol{M} \boldsymbol{A} \boldsymbol{X}$ & \multicolumn{1}{c|}{$\boldsymbol{M I N}$} & \\
\hline OCT & 8,87 & 11,84 & 4,89 & 8,99 & 8,70 & 0,29 \\
\hline NOV & 3,90 & 4,89 & 3,29 & 3,94 & 3,83 & 0,11 \\
\hline DIC & 2,62 & 3,52 & 1,92 & 2,64 & 2,58 & 0,06 \\
\hline ENE & 1,57 & 1,92 & 1,02 & 1,58 & 1,55 & 0,03 \\
\hline FEB & 0,95 & 1,25 & 0,80 & 0,97 & 0,93 & 0,04 \\
\hline MAR & 0,71 & 0,80 & 0,58 & 0,72 & 0,69 & 0,04 \\
\hline ABR & 2,11 & 5,12 & 0,58 & 2,24 & 2,00 & 0,25 \\
\hline MAY & 6,99 & 8,65 & 5,12 & 7,06 & 6,85 & 0,21 \\
\hline JUN & 10,92 & 12,34 & 8,65 & 11,01 & 10,82 & 0,18 \\
\hline
\end{tabular}




\subsection{Ciclos de hielo/deshielo}

Con el objetivo de comprobar el posible cambio de estado del agua en el suelo, como consecuencia de los diferentes regímenes térmicos, se han analizado el número de días que tuvieron la temperatura máxima por debajo de los $0^{\circ} \mathrm{C}$, el número de días que tuvieron la temperatura mínima por encima de los $0^{\circ} \mathrm{C}$ y el número de días donde hubo al menos un cambio entorno a $\operatorname{los} 0^{\circ} \mathrm{C}$, en todos los sensores donde se han obtenido datos (Tablas 26 a 32).

En la estación AIRE, el número de días con ciclos en torno a los $0^{\circ} \mathrm{C}$ es muy elevado durante la temporada nival (Tabla 26), llegando a ocupar en muchos casos la mitad de los días de los meses de diciembre a abril. Únicamente baja los meses más fríos, cuando aumenta los días de helada permanente. Por otro lado, la intensidad del cambio es muy grande, con oscilaciones entre 5 y $7^{\circ} \mathrm{C}$ de media (Tabla 4) y con mínimas entre $\operatorname{los}-3 \mathrm{y}-5^{\circ} \mathrm{C}$ de media (Tabla 3 ).

Tabla 26. Número de días con la temperatura máxima absoluta inferior a $0^{\circ} \mathrm{C}(\mathrm{T}>0)$, días con la temperatura mínima absoluta superior a $0^{\circ} \mathrm{C}(T<0)$ y días con cambios en la temperatura horaria por encima y por debajo de los $0^{\circ} \mathrm{C}(\mathrm{Osci})$, en la estación AIRE. (*) Sólo se emplean los 22 primeros días de junio de 2004. (**) Faltan los 14 primeros días de diciembre de 2005. (***) Faltan los dos últimos días de octubre de 2006.

\begin{tabular}{|c|c|c|c|c|c|c|c|c|c|c|c|c|}
\hline \multicolumn{13}{|c|}{ NÚMERO DE CICLOS EN LA ESTACIÓN: AIRE } \\
\hline \multirow{2}{*}{ MES } & \multicolumn{3}{|c|}{ 2003-04 } & \multicolumn{3}{|c|}{ 2004-05 } & \multicolumn{3}{|c|}{ 2005-06 } & \multicolumn{3}{|c|}{ 2006-07 } \\
\hline & $T>0$ & $T<0$ & Osci & $T>0$ & $T<0$ & Osci & $T>0$ & $T<0$ & Osci & $T>0$ & $\boldsymbol{T}<0$ & Osci \\
\hline OCT & & & & 17 & 0 & 14 & & & & 27 & 0 & $2(* * *)$ \\
\hline $\mathrm{NOV}$ & 15 & 5 & 10 & 18 & 4 & 8 & & & & 19 & 0 & 11 \\
\hline DIC & 4 & 20 & 7 & 2 & 17 & 12 & $0\left({ }^{* *}\right)$ & 6 & 11 & 3 & 9 & 19 \\
\hline ENE & 8 & 8 & 15 & 8 & 8 & 15 & 2 & 19 & 10 & 9 & 9 & 13 \\
\hline FEB & 11 & 10 & 8 & 1 & 15 & 12 & 0 & 9 & 19 & 0 & 4 & 24 \\
\hline MAR & 7 & 6 & 18 & 8 & 6 & 17 & 6 & 3 & 22 & 1 & 10 & 20 \\
\hline ABR & 11 & 7 & 12 & 13 & 5 & 12 & 13 & 0 & 17 & 12 & 6 & 12 \\
\hline MAY & 21 & 3 & 7 & 27 & 1 & 3 & 27 & 0 & 4 & 18 & 1 & 12 \\
\hline JUN & $22(*)$ & 0 & 0 & 30 & 0 & 0 & 29 & 0 & 1 & 29 & 0 & 1 \\
\hline
\end{tabular}

A pesar de los datos mostrados en el AIRE, donde varía entre 80 y 100 el número de ciclos al año, y de la poca importancia de la cubierta nival, en la estación CUMBRE el número de días con ciclos en torno a $\operatorname{los} 0^{\circ} \mathrm{C}$ es muy reducido, precisamente durante la temporada nival. En el caso del sensor de $-10 \mathrm{~cm}$, (Tabla 27), que sería el que debería reflejar más cambios por su carácter superficial y por la escasez de nieve, el número de ciclos en torno a los $0^{\circ} \mathrm{C}$ oscila únicamente entre 10 a 16 al año. Además lo hace con amplitudes mínimas (Tabla 8) y con temperaturas mínimas que descienden como máximo a los $-2^{\circ} \mathrm{C}$ de media, salvo meses excepcionales (Tabla 7). Precisamente, al contrario que en el AIRE, el mayor número de ciclos se da durante el mes de abril, cuando las temperaturas mínimas son muy suaves. Por este motivo, se puede hablar de una acción del hielo/deshielo efectiva únicamente reducida a los meses de primavera, cuando ya ha 
desparecido la nieve. Sin embargo, cuando no hay nieve en el invierno, el número de ciclos disminuye, ya que el suelo permanece helado de forma constante.

Tabla 27. Número de días con la temperatura máxima absoluta inferior a $0^{\circ} \mathrm{C}(T>0)$, días con la temperatura mínima absoluta superior a $0^{\circ} \mathrm{C}(T<0)$ y días con cambios en la temperatura horaria por encima y por debajo de los $0^{\circ} \mathrm{C}(\mathrm{Osci})$, en la estación CUMBRE, en el sensor a-10 cm. (*) Faltan los dos últimos días de junio de 2003. (**) Sólo se registran los primeros 19 días de junio de 2004. (***) Faltan los 15 primeros días de diciembre de 2005.

\begin{tabular}{|c|c|c|c|c|c|c|c|c|c|c|c|c|}
\hline \multicolumn{13}{|c|}{ NÚMERO DE CICLOS EN LA ESTACIÓN: CUMBRE - 10 CM } \\
\hline \multirow[b]{2}{*}{ MES } & \multicolumn{3}{|c|}{ 2002-03 } & \multicolumn{3}{|c|}{ 2003-04 } & \multicolumn{3}{|c|}{ 2004-05 } & \multicolumn{3}{|c|}{ 2005-06 } \\
\hline & $T>0$ & $T<0$ & Osci & $T>0$ & $T<0$ & Osci & $T>0$ & $T<0$ & Osci & $T>0$ & $T<0$ & Osci \\
\hline OCT & & & & & & & 31 & 0 & 0 & & & \\
\hline NOV & & & & 25 & 3 & 2 & 22 & 6 & 2 & & & \\
\hline DIC & 28 & 0 & 3 & 0 & 31 & 0 & 2 & 28 & 1 & 0 & 16 & $0(* * *)$ \\
\hline ENE & 10 & 19 & 2 & 0 & 31 & 0 & 0 & 31 & 0 & 0 & 31 & 0 \\
\hline FEB & 0 & 28 & 0 & 0 & 27 & 2 & 0 & 28 & 0 & 0 & 28 & 0 \\
\hline MAR & 0 & 31 & 0 & 0 & 28 & 3 & 0 & 25 & 6 & 0 & 31 & 0 \\
\hline $\mathrm{ABR}$ & 6 & 19 & 5 & 4 & 19 & 7 & 7 & 13 & 10 & 14 & 0 & 16 \\
\hline MAY & 31 & 0 & 0 & 17 & 12 & 2 & 31 & 0 & 0 & 31 & 0 & 0 \\
\hline JUN & 28 (*) & 0 & 0 & $19(* *)$ & 0 & 0 & 30 & 0 & 0 & 30 & 0 & . \\
\hline
\end{tabular}

Si comparamos estos datos con los del sensor - 60 cm de esta misma estación CUMBRE (Tabla 28), el número de ciclos se reduce de forma considerable. Además, son más numerosos en los días de invierno sin nieve, ya que la intensidad del frío tarda en penetrar a esa profundidad y no está el suelo helado durante varios días, como ocurre en niveles más superficiales. Asimismo, la variación en torno a los $0^{\circ} \mathrm{C}$ es sólo de unas décimas (Tabla 12), con lo que en absoluto se puede hablar de ciclos de hielo/deshielo, al faltar la energía necesaria para producir un cambio de estado en el agua.

Tabla 28. Número de días con la temperatura máxima absoluta inferior a $0^{\circ} \mathrm{C}(T>0)$, días con la temperatura mínima absoluta superior a $0^{\circ} \mathrm{C}(T<0)$ y días con cambios en la temperatura horaria por encima y por debajo de los $0^{\circ} \mathrm{C}(\mathrm{Osci})$, en la estación CUMBRE, en el sensor a -60 cm. (*) Sólo se contabilizan los primeros 19 días de junio de 2004. (**) Faltan los 15 primeros días de diciembre de 2005.

\begin{tabular}{|c|c|c|c|c|c|c|c|c|c|}
\hline \multicolumn{10}{|c|}{ NÚMERO DE CICLOS EN LA ESTACIÓN: CUMBRE - 60 CM } \\
\hline \multirow[b]{2}{*}{ MES } & \multicolumn{3}{|c|}{ 2003-04 } & \multicolumn{3}{|c|}{ 2004-05 } & \multicolumn{3}{|c|}{ 2005-06 } \\
\hline & $T>0$ & $\boldsymbol{T}<\boldsymbol{0}$ & Osci & $T>0$ & $\boldsymbol{T}<\boldsymbol{0}$ & Osci & $T>0$ & $T<0$ & Osci \\
\hline OCT & & & & 31 & 0 & 0 & & & \\
\hline NOV & 30 & 0 & 0 & 30 & 0 & 0 & & & \\
\hline DIC & 31 & 0 & 0 & 31 & 0 & 0 & 8 & 5 & $3(* *)$ \\
\hline ENE & 18 & 12 & 1 & 13 & 13 & 5 & 12 & 10 & 9 \\
\hline FEB & 0 & 29 & 0 & 0 & 28 & 0 & 0 & 28 & 0 \\
\hline MAR & 0 & 31 & 0 & $\underline{0}$ & 31 & 0 & 0 & 31 & 0 \\
\hline $\mathrm{ABR}$ & 0 & 29 & 1 & 4 & 26 & 0 & 16 & 13 & 1 \\
\hline MAY & 31 & 0 & 0 & 31 & 0 & 0 & 31 & 0 & 0 \\
\hline JUN & $19\left(^{*}\right)$ & 0 & 0 & 30 & 0 & 0 & 30 & 0 & 0 \\
\hline
\end{tabular}


En el caso de la estación NIVAL, (Tablas 29 y 30), aunque hay unos pocos cambios en torno a $\operatorname{los} 0^{\circ} \mathrm{C}$, no se puede hablar de ciclos hielo/deshielo, ya que seguramente en ningún momento se produzca la helada del suelo. En el caso del sensor a $-10 \mathrm{~cm}$, las temperaturas mínimas descienden sólo unas décimas, incluso centésimas por debajo de $0^{\circ} \mathrm{C}$ y en el caso del sensor a $-60 \mathrm{~cm}$ ese descenso sólo se produce al final de la temporada estival, después de varios meses con un balance térmico negativo (Tablas 16 y 20). La mínima importancia del descenso térmico y el bajo o casi nulo número de ciclos de hielo/deshielo demuestra que la eficacia de la acción de la helada es insignificante en esta estación.

Tabla 29. Número de días con la temperatura máxima absoluta inferior a $0^{\circ} \mathrm{C}(\mathrm{T}>0)$, días con la temperatura mínima absoluta superior a $0^{\circ} \mathrm{C}(T<0)$ y días con cambios en la temperatura horaria por encima y por debajo de los $0^{\circ} \mathrm{C}(\mathrm{Osci})$, en la estación NIVAL, en el sensor a-10 cm. (*) Faltan los dos últimos días de junio de 2003. (**) Faltan los 14 primeros días de diciembre de 2005.

\begin{tabular}{|c|c|c|c|c|c|c|c|c|c|c|c|c|}
\hline \multicolumn{13}{|c|}{ NÚMERO DE CICLOS EN LA ESTACIÓN: NIVAL - 10 CM } \\
\hline \multirow{2}{*}{ MES } & \multicolumn{3}{|c|}{$2002-03$} & \multicolumn{3}{|c|}{ 2003-04 } & \multicolumn{3}{|c|}{ 2004-05 } & \multicolumn{3}{|c|}{ 2005-06 } \\
\hline & $T>0$ & $T<0$ & Osci & $T>0$ & $T<0$ & Osci & $T>0$ & $T<0$ & Osci & $T>0$ & $T<0$ & Osci \\
\hline OCT & & & & & & & 31 & 0 & 0 & & & \\
\hline NOV & & & & 30 & 0 & 0 & 30 & 0 & 0 & & & \\
\hline DIC & 28 & 0 & 3 & 31 & 0 & 0 & 31 & 0 & 0 & 0 & 4 & $133^{(* *)}$ \\
\hline ENE & 31 & 0 & 0 & 31 & 0 & 0 & 31 & 0 & 0 & 22 & 0 & 9 \\
\hline FEB & 28 & 0 & 0 & 29 & 0 & 0 & 28 & 0 & 0 & 0 & 11 & 17 \\
\hline MAR & 31 & 0 & 0 & 31 & 0 & 0 & 31 & 0 & 0 & 0 & 31 & 0 \\
\hline ABR & 27 & 1 & 2 & 30 & 0 & 0 & 28 & 1 & 1 & 0 & 30 & 0 \\
\hline MAY & 0 & 31 & 0 & 23 & 2 & 6 & 0 & 31 & 0 & 0 & 29 & 2 \\
\hline JUN & $8(*)$ & 19 & 1 & 3 & 26 & 1 & 29 & 0 & 1 & 30 & 0 & 0 \\
\hline
\end{tabular}

Tabla 30. Número de días con la temperatura máxima absoluta inferior a $0^{\circ} \mathrm{C}(\mathrm{T}>0)$, días con la temperatura mínima absoluta superior a $0^{\circ} \mathrm{C}(T<0)$ y días con cambios en la temperatura horaria por encima y por debajo de los $0^{\circ} \mathrm{C}(\mathrm{Osci})$, en la estación NIVAL, en el sensor

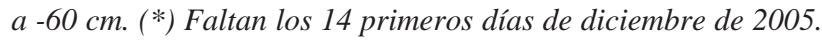

\begin{tabular}{|c|c|c|c|c|c|c|c|c|c|}
\hline \multicolumn{10}{|c|}{ NÚMERO DE CICLOS EN LA ESTACIÓN: NIVAL - 60 CM } \\
\hline \multirow{2}{*}{ MES } & \multicolumn{3}{|c|}{ 2003-04 } & \multicolumn{3}{|c|}{ 2004-05 } & \multicolumn{3}{|c|}{ 2005-06 } \\
\hline & $T>0$ & $\boldsymbol{T}<\boldsymbol{0}$ & Osci & $\boldsymbol{T}>\mathbf{0}$ & $\boldsymbol{T}<\boldsymbol{0}$ & Osci & $\boldsymbol{T}>\boldsymbol{0}$ & $\underline{T}<\boldsymbol{0}$ & Osci \\
\hline OCT & & & & 31 & 0 & 0 & & & \\
\hline NOV & 30 & 0 & 0 & 30 & 0 & 0 & & & \\
\hline DIC & 31 & 0 & 0 & 31 & 0 & 0 & $17\left(^{*}\right)$ & 0 & 0 \\
\hline ENE & 31 & 0 & 0 & 31 & 0 & 0 & 31 & 0 & 0 \\
\hline FEB & 29 & 0 & 0 & 28 & 0 & 0 & 28 & 0 & 0 \\
\hline MAR & 31 & 0 & 0 & 31 & 0 & 0 & 30 & 0 & 1 \\
\hline ABR & 30 & 0 & 0 & 29 & 0 & 1 & 0 & 28 & 2 \\
\hline MAY & 30 & 0 & 1 & 0 & 30 & 1 & 1 & 29 & 1 \\
\hline JUN & 2 & 26 & 2 & 29 & 1 & 0 & 30 & 0 & 0 \\
\hline
\end{tabular}


En el caso de la estación PRONIVAL, (Tablas 31 y 32), el sensor situado a -10 cm mostró un comportamiento muy dependiente con un manto nival, que fue muy irregular durante las distintas temporadas. Durante la temporada 2003/04, con un manto de nieve poco espeso, pero constante, no hay ningún ciclo. En temporadas con períodos sin nieve durante el invierno, el número de ciclos aumenta en esos momentos. Sin embargo, en la primavera, que como ya se ha indicado antes es considerada como la época más adecuada en las áreas pronivales, el número de ciclos es mínimo. Esto se debe a que la nieve, aunque se retira antes que en la estación NIVAL, lo hace bastante después de la estación CUMBRE, ya a finales de abril, cuando las temperaturas mínimas están por encima de los $0^{\circ} \mathrm{C}$. En el caso del sensor a $-80 \mathrm{~cm}$, aunque la temperatura va disminuyendo a lo largo de la temporada nival, no llega a alcanzar los $0^{\circ} \mathrm{C}$ y por tanto no hay ciclos. En definitiva, los resultados indican que en estas áreas pronivales la eficacia de la acción de la helada está también muy atenuada, se reduce al otoño cuando la cubierta nival es escasa y lo hace con una amplitud tan reducida (Tabla 25) que hace dudar que el agua pueda llegar a helarse. Sólo la helada penetra en el suelo los meses sin nieve del invierno, pero esto no supone un elevado número de ciclos, ya que el suelo tiende a permanecer helado de forma constante durante este período seco. En cualquier caso, la helada nunca penetra a los $80 \mathrm{~cm}$ de profundidad.

Tabla 31. Número de días con la temperatura máxima absoluta inferior a $0^{\circ} \mathrm{C}(T>0)$, días con la temperatura mínima absoluta superior a $0^{\circ} \mathrm{C}(T<0)$ y días con cambios en la temperatura horaria por encima y por debajo de los $0^{\circ} \mathrm{C}(\mathrm{Osci})$, en la estación PRONIVAL, en el sensor a-10 cm. (*) Faltan dos días de junio de 2003. (**) Sólo se emplean los 19 primeros días de junio de 2004.

\begin{tabular}{|c|c|c|c|c|c|c|c|c|c|}
\hline \multicolumn{10}{|c|}{ NÚMERO DE CICLOS EN LA ESTACIÓN: PRONIVAL - 10 CM } \\
\hline \multirow{2}{*}{ MES } & \multicolumn{3}{|c|}{$2002-03$} & \multicolumn{3}{|c|}{ 2003-04 } & \multicolumn{3}{|c|}{ 2006-07 } \\
\hline & $T>0$ & $\boldsymbol{T}<\boldsymbol{0}$ & Osci & $T>0$ & $\boldsymbol{T}<\boldsymbol{0}$ & Osci & $\boldsymbol{T}>\boldsymbol{0}$ & $\boldsymbol{T}<\boldsymbol{0}$ & Osci \\
\hline NOV & & & & 30 & 0 & 0 & 30 & 0 & 0 \\
\hline DIC & 20 & 2 & 9 & 31 & 0 & 0 & 15 & 12 & 4 \\
\hline ENE & 6 & 23 & 2 & 31 & 0 & 0 & 23 & 1 & 7 \\
\hline FEB & 0 & 28 & 0 & 29 & 0 & 0 & 28 & 0 & 0 \\
\hline MAR & 0 & 30 & 1 & 31 & 0 & 0 & 31 & 0 & 0 \\
\hline ABR & 5 & 23 & 2 & 30 & 0 & 0 & 30 & 0 & 0 \\
\hline MAY & 31 & 0 & 0 & 31 & 0 & 0 & 31 & 0 & 0 \\
\hline JUN & $28(*)$ & 0 & 0 & $19\left(^{(* *)}\right.$ & 0 & 0 & 30 & 0 & $\underline{0}$ \\
\hline
\end{tabular}


Tabla 32. Número de días con la temperatura máxima absoluta inferior a $0^{\circ} \mathrm{C}(T>0)$,

días con la temperatura mínima absoluta superior a $0^{\circ} \mathrm{C}(T<0)$ y días con cambios en la temperatura horaria por encima y por debajo de los $0^{\circ} \mathrm{C}($ Osci), en la estación PRONIVAL, en el sensor a $-60 \mathrm{~cm}$.

\begin{tabular}{|l|c|c|c|}
\hline \multicolumn{4}{|c|}{$\begin{array}{c}\text { NÚMERO DE CICLOS EN LA } \\
\text { ESTACIÓN: NIVAL - 80 CM }\end{array}$} \\
\hline \multirow{2}{*}{ MES } & \multicolumn{3}{|c|}{$2004-05$} \\
\hline OCT & 31 & 0 & 0 \\
\hline NOV & 30 & 0 & 0 \\
\hline DIC & 31 & 0 & 0 \\
\hline ENE & 31 & 0 & 0 \\
\hline FEB & 28 & 0 & 0 \\
\hline MAR & 31 & 0 & 0 \\
\hline ABR & 30 & 0 & 0 \\
\hline MAY & 31 & 0 & 0 \\
\hline JUN & 30 & 0 & 0 \\
\hline
\end{tabular}

\section{Discusión}

Los resultados obtenidos apoyan la hipótesis de considerar a los neveros de larga duración, situados en montañas templadas, donde no existe permafrost, como un elemento protector del suelo frente a la helada, en la línea de los trabajos publicados previamente sobre el régimen térmico del suelo en estos ámbitos. Así, podemos observar que nuestros resultados son muy semejantes a estudios en neveros situados en áreas bien diferentes, pero siempre donde no existe permafrost, como por ejemplo en Hall (1980), Goodrich (1982), Nyberg (1991) y Kariya (2002). Hecho que acaba definitivamente por demostrar justo lo contrario de la hipótesis inicial de la nivación, que consideraba a los neveros de larga duración como ámbitos donde el suelo helado perduraba mucho más tiempo al año que en las áreas circundantes (Hobbs, 1910; Lewis, 1936, 1939; McCabe, 1939). El régimen bajo los neveros de larga duración es constante y sin grandes variaciones durante las diferentes estaciones, siempre con temperaturas muy próximas a los $0^{\circ} \mathrm{C}$ y con una tendencia a ir perdiendo algunas décimas, incluso centésimas, al mes, hasta alcanzar la temperatura más baja justo antes de la desaparición de la nieve. Las mínimas diferencias entre las temporadas están condicionadas por cuándo se ha iniciado la temporada nival, como recientemente han demostrado Gadek y Leszkiewicz (2009). Si es muy temprano, el calor estival todavía se conserva en el suelo y la temperatura estable inicial comienza desde un nivel más alto, algunas décimas por encima de $0^{\circ} \mathrm{C}$, por lo que tarda más en alcanzar los $0^{\circ} \mathrm{C}$ o incluso no llega a hacerlo en toda la temporada, como, por ejemplo, sucedió en las Guarramillas la temporada 2003/04 (Tablas 13 y 14). Si la cubierta nival se retrasa, la temperatura se estabiliza bajo la nieve muy próxima a los $0^{\circ} \mathrm{C}$, por lo que llega muy pronto por debajo de esa temperatura, aunque sólo sea unas centésimas, como ocurrió en las Guarramillas en la temporada 2005/06 (Tablas 13 y 14). En cualquier caso, el enfriamiento nunca es el suficiente como para que se produzca el congelamiento del agua a una profundidad mínima del suelo. 
Tampoco la acción de la helada tiene importancia en las áreas pronivales en la Sierra de Guadarrama. Los procesos de hielo/deshielo son mínimos o inexistentes, ya que cuando la temperatura del suelo baja por debajo de los $0^{\circ} \mathrm{C}$, lo hace sólo en unas décimas, sin que exista una capacidad real de helar el suelo. El cambio alrededor de los $0^{\circ} \mathrm{C}$ sólo ocurre en otoño y principios del invierno, cuando el retraso de la formación de la cubierta nival es importante, como ocurrió en las Guarramillas en las temporadas 2002/2003 y 2006/07. Mientras que si la llegada de la nieve sigue su ritmo normal, el número de oscilaciones es 0 (Tabla 31). La tradicional hipótesis de la eficacia de la helada en las áreas pronivales en la primavera, justo después del inicio de la fusión de la nieve, considerada de forma continuada (Matthes, 1900; Hobbs, 1910; Grawe, 1936; Lewis, 1936, 1939; McCabe, 1939; Gardener, 1969; Thorn, 1976, 1979, 1988; Hall 1980, 1985, 1993; Thorn y Hall, 1980, 2002, Goodrich, 1982; Nyberg, 1991; Razcokowska, 1995; Christiansen, 1996, 1998; Kariya, 2002; Hall et al., 2002) como el principal agente que genera las habituales coladas de solifluxión pronivales, no se puede aplicar a la Sierra de Guadarrama.

Cuando la nieve se retira en primavera de las áreas pronivales en la Sierra de Guadarrama, la temperatura del aire ya es muy suave y difícilmente baja por debajo de $\operatorname{los} 0^{\circ} \mathrm{C}$. Esto hace que prácticamente no se haya detectado ningún ciclo de hielo/deshielo en el área pronival durante la primavera. Las coladas de solifluxión deben por tanto su actividad, únicamente a la abundancia del agua de deshielo nival y a la preponderancia de materiales finos, características de estos sectores. Seguramente la importancia de la acción de la helada sea fundamental en las coladas de solifluxión pronivales en áreas más frías, donde existe permafrost (múltiples citas desde Ekblaw, 1918, hasta Ishikawa 2003), ya que el nivel impermeable de permafrost sirve de superficie de saturación y despegue en primavera a estas coladas (Ballantyne, 1978). Por el contrario, la helada del suelo nunca llega a más de $1 \mathrm{~m}$ de profundidad en sectores más templados, o con una mayor intensidad de la cubierta nival, donde no existe permafrost, según se ha demostrado recientemente (Zhang et al. 2008). En el caso del Guadarrama, la única temporada donde se han podido obtener datos a una mayor profundidad $(-80 \mathrm{~cm})$, nunca se alcanzaron $\operatorname{los} 0^{\circ} \mathrm{C}$.

La no existencia de suelo helado bajo una cubierta nival de larga duración, hace pensar en la importancia que puede tener la alteración química en esos sectores de un suelo, muy bien hidratado durante la mayor parte del año. En ese sentido, cabría investigar los resultados de esa alteración analizando las aguas pronivales, que han dado resultados muy positivos en otras montañas (Williams, 1949; Darmody et al., 2000, Hall et al., 2002, entre otras muchas publicaciones sobre el tema), y quizás sería ésa la razón fundamental que explicaría por qué hay tanto material fino en el fondo y frente de estos neveros permanentes.

Los únicos sectores donde la acción de la helada es destacable, se sitúan en las crestas más venteadas. En este caso, el régimen de temperatura del suelo cambia completamente de una temporada a otra. Este cambio está en relación con la existencia o no de períodos sin nieve. Si estos períodos son en pleno invierno, el frío penetra con intensidad en el suelo y, cuando la nieve vuelve a cubrir el área, el frío se mantiene en el suelo, cambiando la evolución posterior del régimen térmico del mismo (Gadek y Leszkiewicz, 2009).

La monitorización del régimen de la temperatura del suelo en estos neveros de larga duración y en sus áreas supra y pronivales puede ser un medio efectivo de controlar los 
efectos del cambio climático. Una disminución de la duración de la nieve en el suelo y su retirada temprana en la primavera ha supuesto un claro calentamiento del suelo en áreas árticas (Mellader et al., 2005). También en el ártico, al norte de Alaska, la disminución en el espesor de la nieve, durante los últimos años ha supuesto un calentamiento del suelo, hasta $1,5^{\circ} \mathrm{C}$ al disminuir un $50 \%$ en el espesor de la nieve (Ling y Zhang, 2007). Sin embargo, en las templadas montañas mediterráneas podría suceder lo contrario, ya que la retirada o ausencia de la nieve durante el invierno o principios de la primavera podría suponer, a tenor de los resultados obtenidos, una exposición del suelo a las temperaturas más bajas del aire, lo que no ocurre en la actualidad.

A pesar de coincidir con varios de los modelos de régimen térmico diario propuestos para sectores cimeros de montañas mediterráneas próximas (Vieira et al. 2003), resulta difícil de predecir cuál es su reparto o preponderancia altitudinal, tal y como hacen estos autores. Al menos en la Sierra de Guadarrama, la nieve no sigue unos criterios altitudinales, especialmente en relación con su permanencia (Andrés et al., 2007a y los resultados de este estudio). Sino que precisamente ocurre lo contrario, la mayor parte de las veces, las áreas cimeras son las que menos nieve soportan y ya hemos visto la decisiva importancia que tiene la nieve en el régimen térmico del suelo.

\section{Conclusiones}

Los resultados de la monitorización de la temperatura del aire y del suelo, en tres situaciones claramente diferenciadas por su cubierta nival, indican que la acción de la helada es mucho más reducida en las cumbres de la Sierra de Guadarrama de lo que tradicionalmente se pensaba. Los valores que se obtienen de la temperatura del aire son muy difíciles de extrapolar en el conocimiento del comportamiento térmico del agua en el suelo. Ya a unos pocos centímetros de profundidad en el suelo, y aunque sea con una cubierta mínima e irregular de la nieve, la amplitud térmica se reduce de forma considerable y las temperaturas mínimas son mucho más altas que las del aire. La nieve, aunque tenga un espesor de unos pocos centímetros distorsiona totalmente la amplitud térmica y si supera los $20 \mathrm{~cm}$, la anula. Este hecho hace que durante los meses más fríos de año, la nieve proteja al suelo de la helada. Si no existe, la helada penetra con intensidad en el suelo, pero no le da tiempo a calentarse durante el día, por lo que permanece helado de forma constante. Esta helada únicamente puede ser efectiva a principios de la primavera, pero la nieve está presente entonces en la mayor parte de las áreas cimeras, salvo justo en las crestas. Este es el único momento donde puede haber una acción efectiva de la helada, pero reducida a esos sectores muy venteados.

Cuando la nieve desaparece de las áreas pronivales, ya las temperaturas mínimas no son lo suficientemente bajas como para helar el suelo. La tradicional hipótesis de que es entonces cuando se forman las grandes coladas de gelifluxión pronivales, impulsadas por los procesos de hielo/deshielo, no se puede aplicar a la Sierra de Guadarrama. Deberían llamarse por tanto coladas de solifluxión, ya que el único agente motor es la acción del agua de deshielo que se mezcla con una matriz fina. 
En las áreas nivales no se puede hablar en absoluto de la acción de la helada, ya que esta es inexistente. La temperatura llega a bajar muy superficialmente por debajo de los $0^{\circ} \mathrm{C}$, incluso puede haber un cierto número de días con ciclos en torno a esta temperatura, pero las temperaturas mínimas que se alcanzan suponen únicamente un descenso de unas décimas o incluso centésimas, con lo que difícilmente el agua puede llegar a helarse en ningún momento.

\section{Agradecimientos}

Este trabajo se ha hecho bajo la colaboración y el soporte financiero del Grupo de Investigación UCM: "Geografía Física de Alta Montaña” y varios proyectos consecutivos de la Comunidad de Madrid. Agradecemos a la empresa Retevisión por su ayuda en el mantenimiento de las estaciones meteorológicas.

\section{Referencias bibliográficas}

Andrés, N., PAlacios, D. (2004). Interrelación Nieve / Geomorfología en la Sierra de Guadarrama: altas cuencas del Ventisquero de la Condesa y Valdemartín, Cuadernos de Investigación Geográfica, 30: 83-113.

Andrés, N., García-Romero, A., Muñoz, J., PAlacios, D. (2007a). Characterization of nivation areas in Mediterranean mountains: Manzanares Headvalley, Sierra de Guadarrama (Spain). Zeitschrift für Geomorphologie, 51 Suppl. 2: 91-111.

Andrés, N., Palacios, D., Marcos, F. J. (2007b). Bottom temperature of snow and its geomorphologic significance in Mediterranean Mountains (Sierra de Guadarrama, Spain). Geophysical Research Abstracts, 9: 05639.

Ballantyne, C. K. (1978). The hydrological significance of nivation features in permafrost areas. Geographiska annaler, 60A: 51-54.

Christiansen, H. H. (1996). Nivation forms, processes and sediments in recent and former periglacial areas. Geographica Hafniensia A4, 185 pp., Copenhagen.

Darmody, R. G., Thorn, C. E., Harder, R. L., Schylter, J. P. L., Dixon, J. C. (2000). Weathering implications of water chemistry in an arctic-alpine environment, northern Sweden. Geomorphology, 34: 89-100.

EkBlAw, W. E. (1918). The importance of nivation as an erosive factor, and on soil flow as a transporting agency, in northern Greenland. Proceedings National Academy of Science, 4: 288-293.

GADEK, B., LESZKIEWICZ, J. (2009). Influence of snow cover on ground surface temperature in the zone of sporadic permafrost, Tatra Mountains, Poland and Slovakia. Cold Regions Science and Technology. Doi: 10.1016/j.coldregions.2009.10.004. 
García-Romero, A., Muñoz, J., Andrés, N., Palacios, D. (2009). Relationship between climate change and vegetation distribution in the Mediterranean Mountains: Manzanares Head valley, Sierra de Guadarrama (Central Spain). Climatic Change (aceptado). Doi: 10.1007/s10584-009-9727-7.

GARDENER, J. (1969). Snowpacthes: their influence on mountain wall temperatures and the geomorphologic implications. Geografiska Annaler, 51A: 114120.

Goodrich, E. L. (1982). The influence of snow cover on the ground thermal regime. Canadian Geotechnical Journal, 19: 421-432.

GRAwE, O. R. (1936). Ice as agent of rock weathering: a discussion. Journal of Geology, 44: 173-182.

Hall, K. (1980). Freeze-thaw activity at nivation site in northern Norway. Artic and Alpine Research, 12: 183-194.

HALL, K. (1985). Some observations on ground temperatures and transport processes at a nivation site in northern Norway. Norsk Geografisk Tidsskrift, 39: 27-37.

HALL, K. (1993). Enhanced bedrock weathering in association with late-lying snowpatches: evidence from Livingstone Island. Antarctica. Earth Surface Processes and Landforms 18, 121-129.

Hall, K., Thorn, C. E., Matsuoka, M., Prick, A. (2002). Weathering in cold regions: some thoughts and perspectives. Progress in Physical Geography, 26: 577-603.

Hoввs, W. H. (1910). The cycle of mountain glaciation. The Geographical Journal, 35: $146-163$ у $268-284$.

IsHIKAWA, M. (2003): Thermal regimes at the snow-ground interface and their implications for permafrost investigation. Geomorphology, 52: 105-120.

KARIYA, Y. (2002). Geomorphic processes at a snowpacth hollow on Gassan Volcano, Northern Japan. Permafrost and Periglacial Processes, 13: 107-116.

LEwIS, W. V. (1936). Nivation, River grading and shoreline development in South-East Iceland. Geographical Journal, 88: 431-447.

LEwIS, W. V. (1939). Snow patch erosion in Iceland. Geographical Journal, 94: 153-161.

Ling, F., ZHANG, T. (2007) Modelled impacts of changes in tundra snow thickness on ground thermal regime and heat flow to the atmosphere in Northernmost Alaska. Global and Planetary Change, 57: 235-246.

McCABE, L. H. (1939). Nivation and corrie erosion in West Spitsbergen. Geographical Journal, 94: 447-465.

Marcos, F. J., PAlacios, D. (2004). Efectos de la nieve y la temperatura del suelo en la actividad geomorfológica: primeros resultados de su monitorización en la Sierra de Guadarrama, España. Boletín de la Real Sociedad Española de Historia Natural, 99 (1-4): 25-36. 
Matthes, F. E. (1900). Glacial sculpture of the Bighorn Mountains, Wyoming, U.S. Geological Survey 21st Annual Report 1899-1900: 167-190.

Mellander, P. E., Laudon, H., Bishop, K. (2005). Modelling variability of snow depths and soil temperatures in Scots pine stands. Agricultural and Forest Meteorology, 133: 109-118.

MuÑoz JimÉneZ, J., GarCíA-Romero, A. (2004). Modificaciones climáticas y evolución de la cubierta vegetal en las áreas culminantes de la Sierra de Guadarrama durante la segunda mitad del siglo XX: las altas cuencas del Ventisquero de la Condesa y de Valdemartín, Cuadernos de Investigación Geográfica, 30, págs. 117-146.

Muñoz, J., García, A., Andrés, N., Palacios, D. (2007). La vegetación del Ventisquero de la Condesa (Sierra de Guadarrama, Madrid) y sus condiciones termo-nivales. Boletín de la AGE. 44: 29-52.

NyBERG, R. (1991). Geomorphic processes at snowpatch sites in the Abisko mountains, northern Sweden, Zeitschrift für Geomorphologie N.F. 35(3): 321-343.

RaczKowska, Z. (1995). Nivation in the High Tatras, Poland. Geografiska Annaler 77A (4): 251-258.

RozeT, M. (1855). Notes on the differences of temperature between the air, the ground under snow, and the ground from which the snow has been removed. Journal of the Franklin Institute, 59 (4): 270-271.

Thorn, C. E. (1976). Quantitative evaluation of nivation in the Colorado Front Range, Geological Society of America Bulletin, 87: 1.169-1.178.

THORN, C. E. (1979). Ground temperatures and surficial transport in colluvium during snowpatch; Colorado Front Range. Earth Surface Processes 4, 211-228.

Thorn, C. E. (1988). Nivation: a Gemorphic Chimera. En: Advances in Periglacial Geomorphology (Clark, M.J., Ed.). John Wiley \& Sons Ltd, pp. 3-31, Chichister.

ThORN, C. E., HALl, K. (1980). Nivation: an arctic-alpine comparison and reappraisal. Journal of Glaciology, 25: 109-124.

Thorn, C. E., Hall, K. (2002). Nivation and cryoplanation: the case for scrutiny and integration. Progress in Physical Geography, 26(4): 633-550.

Vieira, G., Mora, C., Ramos, M. (2003). Ground temperature regimes and geomorphological implications in a Mediterranean mountain (Serra da Estrela, Portugal). Geomorphology, 52 (2003) 57-72.

Williams, J. E. (1949). Chemical weathering at low temperaturas. Geographical Review, 1: $129-135$

ZhANG, Y., WANG, Y., BARR A. G., Black ,T. A. (2008). Impact of snow cover on soil temperature and its simulation in a boreal aspen forest. Cold Regions Science and Technology, 52: 355-370. 